\title{
Peridynamic Investigation of the Effect of Porosity on Fatigue Nucleation for Additively Manufactured Titanium Alloy Ti6Al4V
}

\author{
Olena Karpenko, Selda Oterkus*, Erkan Oterkus,
}

Department of Naval Architecture, Ocean and Marine Engineering, University of Strathclyde, 100 Montrose Street, Glasgow, United Kingdom

*Corresponding author: selda.oterkus@strath.ac.uk

Keywords: Peridynamics; Additive manufacturing; porosity defects; fatigue life prediction;

\begin{abstract}
Additive manufacturing (AM) has enabled the fabrication of complex geometries directly from the design data and gained a lot of interest. However, AM products can contain pores, which can significantly reduce fatigue life. The main focus of this work is to evaluate the effect of the pores in Ti6Al4V dog-bone samples, produced by AM, and propose a methodology to estimate the fatigue life reduction due to the internal pores. The bond-based Peridynamics (PD) fatigue model was utilised to analyse the fatigue life of the defect-free sample with the calibrated PD parameters. Moreover, a numerical model is developed to investigate two types of porosities in a system. The application of the PD model showed a capability of crack nucleation prediction for the titanium alloy samples under cycling loading. The predicted results are compared with experimental data using the stress-life (S-N) curve. Furthermore, this paper presents a numerical approach to assess the influence of the pore location and size on the fatigue life of Ti6Al4V. The PD predictions indicated the critical pore characteristics and the applicability of the developed PD model on samples with low porosity for high cycle fatigue-loaded applications.
\end{abstract}

\section{Introduction}

Additive manufacturing (AM), or known as 3D printing technology, is a subject of undergoing intense research and development studies in the field of Metal Rapid Prototyping (MRP). 3D printed structures are widely used in aerospace, automotive and biomedical industries [1-4], and, nowadays, its application is extended to the marine industry $[5,6]$.

The rapid increase of $A M$ in industrial applications provides flexibility in designing and manufacturing of complex metal structures involving titanium, stainless steel, nickel and aluminium alloys [7]. Moreover, the AM process of building layer by layer metal components according to the designed 3D model [3], is increasing production 
efficiency and product customisation. Titanium alloys Ti6Al4V receive particular interest in most of the fields due to its high specific strength and corrosion resistance. Therefore, some experimental studies showed the existence of the process-induced defects and the differences in the product quality of the structures produced by additive layer manufacturing (ALM), which can have an influence on the fatigue performance of the AM parts [8-11]. Firstly, the variations in the microstructure and the material properties (layer quality) were reported [12,13]. The changes in the overall composition of the alloy are due to vaporisation of the elements when the temperatures in the molten pool are very high [14]. Moreover, the deposition process has to be carefully controlled as it could lead to the entrapped gases or vapours in the material resulting in microscopic spherical pores [7]. Secondly, recent studies on wire + arc AM (WAAM) and selective laser melting (SLM), using in-situ X-ray computed tomography (CT) scanning, evidenced the presence of the pores in the structures. The porosity volumes for each of the methods vary and are reported as $0.01-0.04 \%$ for WAAM [8] and $0.08-0.2 \%$ for SLM [15]. The variations of the porosity inclusions are the cause of cooling rates and temperatures of the AM process [16]. It is commonly observed that pores are in the regions of non-melted powder for SLM [17] and when the structure is manufactured with a contaminated wire during WAAM [10]. With this respect, it is essential to understand the cause of the mechanical property variations of the metal alloys produced by AM and the effect of porosities on Ti6Al4V fatigue properties.

The Ti6Al4V fatigue properties have significantly scattered experimental data. Besides, the pores in the structures have a significant influence on the fatigue life of AM samples. Experimental studies on Ti6Al4V fatigue properties showed that the crack nucleation starts at the process-induced pore and the lifetime to failure depends on location, size and pore volume [11,18-21]. Moreover, indicating the types of pores according to their location as on surface, subsurface and internal pores, the surface pores have a higher impact on the Ti6Al4V fatigue properties [8]. The surface roughness is the major drawback of the AM process, which limits fatigue performance. The studies $[22,23]$ showed that the post-processing of the samples increases the fatigue performance where the smoother surface brings higher fatigue life. On the other hand, the surface pores or surface roughness can be removed by postprocessing techniques, like chemical polishing, machining, grinding and peening. With the use of post-processing techniques and the carefully controlled processing conditions, input parameters can avoid the surface pores and subsurface pores, located in the vicinity of the free surface, specified as the most critical one $[10,24,25]$. 
It is important to note that crack initiation occurs at the areas with a more considerable amount of porosity or the pores located close to the free surface. Multiple tests $[9,10,26,27]$ indicated that the sub-surface pores are the dominant factor of limiting fatigue life. Also, the researchers identified the challenges for determining the AM Ti6Al4V fatigue properties, as layer by layer manufacturing results in heterogeneous microstructure and porosity defects distributions.

Ti6Al4V porosities have a direct impact on mechanical behaviour [28]. Also, porosity affects the elastic properties of the material, and the proposed empirical and semi-empirical expressions [29-37] relate Young's modulus to the total porosity of the sample. The proposed analytical solutions include the characterisation of the pore sizes, ratio, orientation, fraction, etc. However, the proposed equations have limitations and include assumptions about the real microstructure, including the parameters, which has to be measured experimentally for each type of material. The proposed analytical approaches showed a good fit to experimental values for brittle fracture with the main focus on the prediction of the elastic properties of porous materials. While quasi-static properties are essential for initial evaluation of the material, the prediction of the fatigue life of the material is more crucial.

The predictive model, based on the defect tolerant design approach, such as ElHaddad's [38], is widely used to predict the fatigue limit of AM materials. In this model, the defect is assumed as a crack and later Murakami and Endo [39] introduced a new parameter based on the projected area of the pore expressing the fatigue limit as a function of the porosity size and location. In addition, Beretta and Romano [40] adapted Murakami's parameter in El-Haddad's model for calculating the fatigue limit of the samples including pores. The analytical approaches [38-40] showed reasonable estimation of the endurance limit in comparison to the fatigue test of AM samples $[9,10,20,41]$, but in some cases can give conservative predictions. The numerical crack nucleation models can be developed for more accurate predictions of fatigue performance. The studies [11,42] are modelling an individual pore using a finite element method to compute the stress concentration factor. The performed static analysis with a defined ratio of pore diameter to its distance to the free edge [42] concluded that the stress concentration factor is higher for the pores located close to the free surface. Instead, in [11], the porosity defects of different shapes are identified by scanning the samples of SLM Ti6AI4V and evaluating the stress concentration factor by FEA. The fatigue nucleation is predicted at the pore by using the stress-strain response of the FE model. Nonetheless, the following numerical studies focused on a single pore and did not include the global interaction on the multiple pores. 
This study is focused on the effect of the pores in the AM Ti6Al4V samples by taking into account the porosity of the material and pore distributions. To analyse the influence of the pores on the high cycle fatigue (HCF) crack initiation, the "bond-based" peridynamics (PD) is used. The PD model, developed by Silling in [43], is successfully applied to brittle facture problems on crack nucleation and propagation [44,45], as well as on micro-crack interaction problems [46,47]. Furthermore, Oterkus et al. [48] proposed a PD fatigue model which applies only on crack growth and Silling and Askari [49] introduced a "bond-based" PD approach to treat crack nucleation and propagation under the cycling loading. The applicability of the model [49] is shown on fatigue crack propagation problems in metals [50] and composites [51].

The predictive HCF nucleation model [49] is developed to investigate the influence of changes in the porosity of the AM Ti6Al4V. In Section 2, the bond-based PD model is briefly introduced for fatigue nucleation. Considering the duration of the fatigue simulations, the direct solution with stabilised biconjugate gradients method (BiCG) is used to improve the computational efficiency. In Section 3, the defect-free titanium alloy is used to calibrate PD parameters using the tests data from [8], and the convergence study of fatigue nucleation in a dog-bone specimen is performed. In Section 4, the fatigue nucleation analysis is performed for AM Ti6Al4V samples with two different porosity levels. The porosity in fatigue models is introduced with gamma distributions in order to reach the required porosity levels. Firstly, the porosities in the fatigue model are implemented with a "concentration-dependent damage" (CDD) [52] approach and fatigue life were predicted. Secondly, new peridynamic porosity model is proposed to model the low porosity in the samples, which improves the prediction accuracy of the fatigue nucleation. Both of the models are validated against the tests. Lastly, the sensitivity study is performed on the reduction of material life with the located single pore and changing its diameter and location. Finally, the discussions are given in Section 5 and followed by conclusions.

\section{Method}

\subsection{Peridynamic model for brittle damage}

The bond-based peridynamics (PD) introduced by Silling [43] is a reformulation of the fundamental equations of continuum mechanics equations which are particularly suitable to solve problems including discontinuities. Peridynamics uses integrodifferential equations instead of partial differential equations as in classical continuum 
mechanics. This widens the possibility of solving fracture mechanics problems including cracks initiation and propagation [53].

The peridynamic equation of motion can be written in the form of the integrodifferential equation as:

$$
\rho(\boldsymbol{x}) \ddot{\boldsymbol{u}}(\boldsymbol{x}, t)=\int_{H_{x}} \boldsymbol{f}\left(\boldsymbol{u}\left(\boldsymbol{x}^{\prime}, t\right)-\boldsymbol{u}(\boldsymbol{x}, t), \boldsymbol{x}^{\prime}-\boldsymbol{x}\right) d V_{x^{\prime}}+\boldsymbol{b}(\boldsymbol{x}, t)
$$

which can be discretised as

$$
\rho_{(i)} \ddot{\boldsymbol{u}}_{(i)}=\sum_{j=1}^{N} \boldsymbol{f}_{(i)(j)} V_{(j)}+\boldsymbol{b}_{(i)}
$$

from which the acceleration $\ddot{\boldsymbol{u}}_{(i)}$ of the material point $i$ at time $t$ can be obtained. Each material point $i$ interacts with other material points $j$ within its horizon $H_{x}$ with a total number of $N$ family members, as shown in Fig. 1 . The coordinates of a material point are represented as $\boldsymbol{x}$ with the incremental volume $V . \boldsymbol{u}, \boldsymbol{b}$ and $\rho$ denote displacement vector field, body load and mass density of the material point, respectively.

In the bond-based Peridynamics, interacting material points can exert forces on each other with equal magnitudes [54] and for the interacting material points $i$ and $j$, peridynamic pairwise force densities can be defined as [55]:

$$
f_{(i)(j)}=4 \delta b s_{(i)(j)} \frac{\boldsymbol{y}_{(j)}-\boldsymbol{y}_{(i)}}{\left|\boldsymbol{y}_{(j)}-\boldsymbol{y}_{(i)}\right|}
$$

where $\delta$ is the horizon size, $b$ is the peridynamic parameter and $s$ is the stretch between material points. Once a material point displaces to a new location as a result of deformation of the structure, its new location is specified as $y$ in the deformed configuration, as shown in Fig. 2.

Peridynamic parameter $b$ can be related to the material constant of classical continuum mechanics by equating strain energy density of a material point inside a body subjected to isotropic expansion and simple shear loading conditions calculated from classical continuum mechanics and peridynamics [54]. In this paper, 2D-model with plane stress conditions is used, where the plate is discretised with a single layer of material points in the thickness direction. Due to this, the PD parameter is expressed in terms of shear modulus, $\mu$, thickness, $h$, and horizon size, $\delta$, for a 2-Dimensional problem:

$$
b=\frac{6 \mu}{\pi h \delta^{4}}
$$

The stretch between material points $i$ and $j$ can be expressed as 


$$
s_{(i)(j)}=\frac{\left|\boldsymbol{y}_{(j)}-\boldsymbol{y}_{(i)}\right|-\left|\boldsymbol{x}_{(j)}-\boldsymbol{x}_{(i)}\right|}{\left|\boldsymbol{x}_{(j)}-\boldsymbol{x}_{(i)}\right|}
$$

\subsection{Peridynamic model for fatigue damage}

The first peridynamic fatigue model was proposed in [48], which applies only to the crack growth phase. Instead, the formulation in [49] describes the possibilities to deal with three phases of fatigue: crack nucleation, crack growth and final failure. The damage variable called the "remaining life" was introduced, which is degrading over the fatigue cycles. Each bond of the material points interactions in the PD model is identified by initial remaining life $\lambda(0)=1$ and the bond is breaking irreversibly when $\lambda(N) \leq 0$. The irreversible bond breakage for fatigue cracking can be implemented by the failure parameter [56] in the equation of motion Eq. (2), which includes a historydependent scalar-valued function $\mu$ to represent broken interactions (bonds) between material points:

$$
\mu\left(\boldsymbol{x}_{(j)}-\boldsymbol{x}_{(i)}, N\right)=\left\{\begin{array}{cc}
1 & \text { if } \lambda(N)>0 \text { for all } 1<N^{\prime}<N \\
0 & \text { otherwise }
\end{array}\right.
$$

To monitor the accumulated damage of the bonds over the loading cycles $N$, the local damage of each material point $i$ is defined as:

$$
\varphi\left(\boldsymbol{x}_{(i)}, N\right)=1-\frac{\sum_{i=1}^{N} \mu\left(\boldsymbol{x}_{(j)}-\boldsymbol{x}_{(i)}, N\right) V_{(j)}}{\sum_{i=1}^{N} V_{(j)}}
$$

The introduced evolution law in [49] for the remaining life of the bond is:

$$
\frac{d \lambda(N)}{d N}=-A \varepsilon^{m}
$$

where $\varepsilon$ is the cyclic bond strain for the sample, which is under two extremes of the loading conditions. The application of each extreme in the model is resulting in maximum and minimum bond stretches between material points, so the bond strain is defined as:

$$
\varepsilon=\left|s^{\max }-s^{\min }\right|=\left|(1-R) s^{\max }\right|
$$

The material behaviour under two extremes is assumed to be linear, so the cyclic bond strain is expressed in Eq. (9) by $s^{\max }$ and load ratio $R=s^{\min } / s^{\max }$.

The parameters $A$ and $m$ in Eq. (8) are the material constants that have to be calibrated using the S-N curve data for the crack initiation phase and with Paris law for fatigue crack growth. 
For the crack nucleation phase, the parameters $A$ and $m$ are denoted as $A_{1}$ and $m_{1}$, and have to be calibrated from experimental data. Because the initial remaining life of each bond is $\lambda_{1}(0)=1$, integrating Eq. (8) leads to:

$$
A_{1} \varepsilon^{m_{1}} N_{1}=1
$$

This means that the crack nucleation occurs at:

$$
N_{1}=\frac{1}{A_{1} \varepsilon^{m_{1}}}
$$

Presenting Eq. (11) in a log-log scale, the $A_{1}$ and $m_{1}$ can be determined by a linear fit to experimental data presented by strain-life relation in log-log scale. The obtained parameters of $A_{1}$ and $m_{1}$ are used for static solutions to calculate the fatigue life of the sample at the nucleation phase. The calculations will continue until the local damage is $\varphi<0.4$, and fatigue damage will move from phase I (crack nucleation) to phase II (crack growth).

The studies in the present work focus only on crack nucleation phase, so the phase II is not presented.

\subsection{Direct Peridynamic solver for fatigue damage}

Fatigue analysis includes multiple static simulations. Most of the studies on PD static or quasi-static solutions are using explicit time integration. Moreover, Eq. (2) is introduced in the dynamic form, and a commonly used technique, as Adaptive Dynamic Relaxation (ADR)[53], is implemented in PD theory to solve static or quasistatic problems. Using the explicit method, the convergence of the static solution can be time-consuming, as too many time steps are required to reach a stable solution. Such a method for fatigue analysis can be very costly, and some studies are performed using an implicit solution [50]. In the current work, the direct method [57] is used where PD force function for a 2-D problem is expressed in the matrix form:

$$
\left\{\begin{array}{c}
f_{x} \\
f_{y}
\end{array}\right\}=\frac{4 \delta b}{|\xi|^{3}}\left[\begin{array}{ll}
\xi_{x} \xi_{x} & \xi_{x} \xi_{y} \\
\xi_{y} \xi_{x} & \xi_{y} \xi_{y}
\end{array}\right]\left\{\begin{array}{c}
\eta_{x} \\
\eta_{y}
\end{array}\right\}
$$

where $\xi$ is the relative position between material points, $\boldsymbol{\xi}=\boldsymbol{x}^{\prime}-\boldsymbol{x}, \boldsymbol{\eta}$ is the relative displacement between material points, $\boldsymbol{\eta}=\boldsymbol{u}\left(\boldsymbol{x}^{\prime}, t\right)-\boldsymbol{u}(\boldsymbol{x}, t)$ and $\xi_{x}, \xi_{y}$ are the reference lengths in $x$ and $y$ directions.

In order to solve the static problem using the direct method, the acceleration term $\ddot{u}$ is omitted from the equation of motion, and the equation will have the following form:

$$
\sum_{j=1}^{N} \boldsymbol{f}_{(i)(j)} V_{(j)}+\boldsymbol{b}_{(i)}=0
$$


Note that Eq. (13) contains a local stiffness for each material point, interacting with other material points. The combination of local stiffness matrices is resulting in the global static PD equation written in the matrix form as:

$$
\boldsymbol{K} \boldsymbol{U}=\boldsymbol{B}
$$

where $\boldsymbol{K}, \boldsymbol{U}$ and $\boldsymbol{B}$ are the global stiffness matrix, the material points' displacement and the body load matrices. Eq. (14) is solved to find the displacements of each material point, by calculating the inverse stiffness matrix $\boldsymbol{K}^{-1}$. For the models with fine discretisation, the global stiffness matrix can be very massive, and it is time-consuming to calculate the $K^{-1}$. To overcome the following issue, the stabilised biconjugate gradients method (BiCG) is used. BiCG improves the capabilities of the PD solver by making it faster and smoother convergence. For all the presented simulations the BiCG method is used with the convergence tolerance of $10^{-6}$ and the maximum number of iterations of 5000 .

\section{The fatigue life of defect-free Ti6Al4V Titanium alloy}

The AM Ti6Al4V got attention in multiple fields due to its high strength to weight ratio, and a lot of studies are focused on Ti6Al4V fatigue properties. The investigations showed that the factors, like AM processing parameters, the geometry and the surrounding environment, have a direct impact on Ti6Al4V mechanical properties [58]. In the presented studies, the AM processing parameter variations and its processingproperty relationships are not taken into account, as well as the potential anisotropy of mechanical properties. Therefore, the resulting process-induced pores in the dogbone samples and their effect on the Ti6Al4V fatigue properties are investigated. In this respect, the defect-free Ti6AI4V sample with isotropic properties is selected as a reference specimen, and different methods of pores implementation in PD model are presented with its effect on the fatigue performance. In Sect. 3, the PD defect-free high cycle fatigue nucleation model set up is presented.

\subsection{Properties of PD model}

The 2D peridynamic fatigue model is considered for a dog-bone plate with a total length of $L=30 \times 10^{-3} \mathrm{~m}$, a width of $W=10^{-2} \mathrm{~m}$ and $W_{\text {in }}=5 \times 10^{-3} \mathrm{~m}$, the thickness of $h=5 \times 10^{-3} \mathrm{~m}$ shown in Fig. 3a. The following homogeneous material properties of the plate are specified: Young's modulus $E=110 \mathrm{GPa}$ and Poisson's ratio $v=1 / 3$. 
Using the test data [8] for the defect-free sample, the calibration of the parameters $A_{1}=2.9 \times 10^{24}$ and $m_{1}=13.56$ are utilized for PD numerical model [49]. The specimen is under high cycle fatigue with the load ratio of $R=0.1$ and the stress amplitudes of $\sigma_{a}=360,316$ and $270 \mathrm{MPa}$ are considered. A static loading, using the direct method described in Sect. 2, is applied as a body force density, $b_{y}$ (Fig. 3b):

$$
b_{y}=\frac{\sigma_{y} W h}{\Delta V_{\Delta}}
$$

where $\Delta \mathrm{V}_{\Delta}$ is the volume of the boundary layer, and $\sigma_{y}$ is a tension loading.

$$
\sigma_{y}=\sigma_{y}^{\max }=\frac{2 \sigma_{a}}{1-R}, \sigma_{y}=\sigma_{y}^{\min }=\sigma_{y}^{\max } R .
$$

Please note that the no-damage zone is introduced in all numerical models in order to avoid the crack initiation in the area of the abrupt changes in the crosssectional area. In that matter, the accumulated damage of the bonds is allowed only for the points located in the midsection of the sample $\left(-2.5 \times 10^{-3} \mathrm{~m} \leq y \leq\right.$ $\left.2.5 \times 10^{-3} \mathrm{~m}\right)$.

\subsection{Numerical study of convergence}

Three types of convergence studies are identified in [59]. In this work, the $\delta$ convergence study is performed for a fixed horizon factor $m=3$, where $\delta=m \Delta x$. The problem set up is stated in Sect. 3.1 and the dog-bone plate is under the stress amplitude of $\sigma_{a}=360 \mathrm{MPa}$. Three different horizon sizes $\delta$ with corresponding uniform grid spacing and the total number of material points of $\Delta x=0.2 \times$ $10^{-3} \mathrm{~m}$ (4656 nodes),$\quad \Delta x=0.15 \times 10^{-3} \mathrm{~m}$ (8352 nodes),$\quad \Delta x=0.1 \times$ $10^{-3} \mathrm{~m}$ (18784 nodes) are selected. The parameters $A_{1}$ and $m_{1}$ are kept constant for $\delta$-convergence studies, as they are obtained from the test data and are not dependent on the horizon size. The focus of $\delta$ - convergence is to observe the crack nucleation phase in a specimen and the fatigue life. The PD simulations are stopped when the nucleation of the damage occurs, meaning that the local damage is $\varphi \geq 0.4$. Fig. 4 shows the comparison of damage plots, where the fatigue damage is at the midplanes of the specimen. With the increased number of material points within the sample, the damaged area is smaller, but there is no major impact on the fatigue life, where the difference in life predictions is $<1 \%$. Considering that the outcome of this study showed quite close results for all three horizon sizes, PD models with $\delta=0.45 \times 10^{-3} \mathrm{~m}$ and $m=3$ will be utilised for numerical efficiency. 


\subsection{Crack nucleation prediction}

As observed in [8], the type of wire used for WAAM of titanium alloy resulted in different porosity in the samples and moreover, in the variations of the fatigue life. Due to this, the reference PD model is set up for a defect-free Ti6Al4V specimen, and Fig. 5 shows the S-N data of the numerical results in comparison with the experimental data [8]. All the HCF nucleation life predictions are presented in logarithmic scale. It can be seen in Fig. 5 that the predicted crack initiation life for three different amplitudes $\sigma_{a}=360,316$ and $270 \mathrm{MPa}$ are in good agreement with the experimental data and the developed numerical model is used as the reference one for the porosity implementations in the next presented studies.

\section{The fatigue life of the sample with micro-pores}

In this section, the "concentration-dependent damage" (CDD) [52] approach of porosity implementation is utilised in Peridynamic fatigue model. Then, a new method is introduced for pores representation in the numerical PD fatigue model.

\subsection{Numerical model assumptions}

The material exposed to cyclic loading undergoes different phases with a particular behaviour. Each of the phases is load-dependent, where the damage nucleation in the material is initiated from the fractions of $10 \mathrm{~nm}$ leading to the development of micro-cracks in the crystallographic planes initiated by the shearing stress. During the low-cycle fatigue (LCF), the material is exposed to the plastic deformations due to the large and continuous plastic slips in the grains. In the HCF the shear stress is also activating the localised plastic slips in some crystals and on a surface of the material, where the damage nucleates in the form of pores or cavities. The crack nucleation is a complex process which is dependent on the loading and the material composition, where a very small fraction can initiate the material damage and reduce the fatigue life. The damage on sub-microscopic level initiated with slip bands was investigated in theoretical studies [60] and, the studies [61,62] of fatigue behaviour of the material with different inclusion sizes, shapes and location indicated the stress concentrations at inclusion apex, where the inclusions with dimensions of $d_{p}>10 \mu m$ at sub-surface level originated the crack nucleation. Even perfectly round defects artificially induced in the sample had an effect on crack nucleation and the loss of fracture ductility during LCF investigation [63]. The in-situ X-ray computed tomography (CT) scanning of AM materials, evidenced the presence of the spherical 
pores in the structures, which were immediately below the surface of the sample and/or inside the sample, and the damage nucleation occurred directly from the pore. Due to this, the assumptions were put in place in the presented PD model, where the evolution of the crack nucleation is starting at the pore and not at plastic slips, so the pore is always in the elastic domain and no plastic flow occurs.

\subsection{Problem setup}

All the presented numerical studies are performed on the Ti6Al4V dog-bone specimen (Fig. 3) with the geometrical and material properties stated in Sect. 3. Moreover, the PD numerical model from Sect. 3 is selected as a reference defect-free model. The specimens are under HCF with the load ratio of $R=0.1$ and the stress amplitudes of $\sigma_{a}=360,316$ and $270 \mathrm{MPa}$. For each load, a number of simulations are performed with varying pore distributions within the midsection of the sample. As the current interest is at nucleation of the damage, the solver is stopped when local damage is $\varphi \geq 0.4$.

The pores are randomly distributed in the sample as schematically shown in Fig. 6. Gamma distribution with the shape parameter of $a=2$ is selected. The scale parameter and the total number of pores $N_{p_{t o t}}$ are implemented with variations in order to achieve the desired porosity ratio in a sample. The flow-chart of porosity evaluation and pore assignment is shown in Fig. 7. After the desired total porosity is reached, a set of pores $N_{p_{t o t}}$ with the optimal pore diameters are randomly allocated in the sample.

To evaluate the fraction of void space in the material, the following porosity ratio is used:

$$
P=\frac{V_{p}}{V_{T}}
$$

where $V_{p}$ is the volume of void-space and $V_{T}$ is the bulk volume of material.

Two types of porosities are selected for verification study of PD numerical model with the tests [10]. During the experiments and analysis of the additively manufactured samples [10], the porosity specimens are deposited with contaminated wire, and the porosities of $P=0.01 \%$ and $P=0.04 \%$ are identified. Moreover, looking over the microstructure of the specimens, it is observed that the specimens with porosity of $P=$ $0.01 \%$ include pores with diameters below $100 \mu \mathrm{m}$. Having this in mind, two types of simulations are performed using Gamma distribution of the pores in order to reach the 
optimal pore diameters with the porosities of $P=0.01 \%$ and $P=0.04 \%$. Fig. 8 shows the examples of the pore distributions.

\section{3 'Concentration-dependent damage' peridynamic model}

The CDD in the PD model is representing the porosity in the system with the predamage initiation. The following approach has been successfully utilised for the damage occurred by corrosion [52] and later on implemented in elastic porous materials like glass and rock [64], which are subjected to force pulses to evaluate the wave propagation. In the current study, the CDD model is used to initiate the porosity in the dog-bone specimen (Fig. 3) and to evaluate the influence of the pores on the fatigue life in HCF problems.

In peridynamics, the failure in the material is calculated as $N_{b} / N_{T}$, where the ratio between the number of broken bonds is $N_{b}$ and the total number of the bonds is $N_{T}$. To implement pore in the system, we assume that the damage index at a point is linearly dependent on its porosity, where the porosity ratio for each material point $x_{i}$ can be represented as:

$$
P\left(x_{(i)}\right)=\frac{N_{b(i)}}{N_{T(i)}}
$$

and the total porosity of the sample is evaluated in the following form:

$$
P=\frac{\sum_{i=1}^{N} \frac{N_{b(i)}}{N_{T(i)}} V_{(i)}}{\sum_{i=1}^{N} V_{(i)}}
$$

This means that initial damage is initiated in the model and implemented by breaking bonds in order to match the specified porosity. Note that in the current study, the interest is in low porosity of the metals and not in the porous materials.

Using the CDD model, the number of broken bonds have to be evaluated for each pore. Combining Eq. (17) and Eq. (18) the number of pre-broken bonds for each material point is considered as:

$$
N_{b(i)}=\frac{V_{p(i)}}{V_{T(i)}} N_{T(i)}
$$

where $V_{p(i)}$ is the volume of the pore assigned for the material point $x_{i}, V_{T(i)}$ and $N_{T(i)}$ are the bulk volume and the total number of bonds of the material point, respectively. All the pores are treated as spherical, and for a $2 \mathrm{D}$ sample, the volume of the pore with a diameter $d_{p}$ is evaluated as $V_{p}=\frac{4}{3} \pi\left(\frac{d_{p}}{2}\right)^{3}$. Utilising Eq. (20) for analysis of the number of the pre-broken bonds to be assigned in the selected material points with the allocated pore of diameter $d_{p} \leq 400 \mu m$, the relationship between $N_{b(i)}$ and $d_{p(i)}$ 
can be evaluated as shown in Fig. 9. It should be noted that the bonds can be fully and partially broken. For example, if the pore diameter of $d_{p}=300 \mu \mathrm{m}$ is allocated for material point, then three bonds are fully broken with $\mu=0$ and one partially broken with $\mu=0.519$ based on the pore diameter and the number of broken bonds. This means that a history-dependent scalar-valued function $\mu$ in Eq. (6) for pre-broken interactions (at cycle $N=0$ ) between material points will be $\mu=1$ if $d_{p}=0$ and $\mu<1$ for $d_{p}>0$. Due to the fact that the scanned samples in the experimental studies [10] showed porosities of $d_{p}<400 \mu m$, the number of pre-broken bonds per node is $N_{b}<$ 9 (Fig. 9). Due to the random breakage of bonds in the selected material points, the bonds connecting two material points with the pores will stay intact. It means that if the material point $x_{i}$ and material point $x_{j}$ are selected for the representation of the pore, then the bond $x_{i}-x_{j}$ will not be broken, and the bond-breakage moves to the next bond of the family members of point $x_{i}$. With the stated bond-breakage system, the assumptions of the "isotropic" pore shapes and distribution, and the linear dependency of local damage on its porosity, the local damage of each material point is evaluated by Eq. (7).

The numerical results of the CDD model implementation of porosities in the dogbone sample have shown that the damage is starting at the pore located close to the edge of the sample. The bigger the pore and closer it is to the edge, the faster the damage initiates. Fig. 10 represents the damage maps of three solutions under different loading conditions with the porosity of $P=0.04 \%$ in the samples. Fig. 11 shows the numerical results and comparison to experiments [10]. For the lower porosities (Fig. 11a), with the implemented CDD method, the fatigue life $N_{f}$ is quite close to experimental data. However, for the porosity of $P=0.04 \%$, the fatigue life is overpredicted (Fig. 11b).

The presence of the pores with the implemented CDD model results in local stress concentrations at the location of the pore with the crack initiation at the pore. In reality, a similar trend of pore initiation is noticed during the experiments on AM samples [65]. Therefore, the employment of the CDD method in PD HCF fatigue model did not show good applicability on fatigue problems, due to the different nature of the fatigue crack nucleation and the processes developing in the material under the cycling loading. Due to this, additional porosity models are investigated with the effect of the pores on fatigue limit. 


\subsection{New peridynamic porosity model}

Evaluating the fatigue life of the structures, porosity defects has a noticeable effect on fatigue strength and multiple models, summarised and classified in [66], were proposed to predict the impact of the small cracks or inclusions on fatigue life. El Haddad et al. [38] presented a model in Eq. (21) in order to use the LEFM theory for short cracks and introduced the fictitious crack length $l_{0}$ in Eq. (22), which is increasing with a very short length of the crack.

$$
\begin{aligned}
& \Delta K=\Delta \sigma \sqrt{\pi\left(l+l_{0}\right)} \\
& l_{0}=\frac{1}{\pi}\left(\frac{\Delta K_{t h}}{\Delta \sigma_{e}}\right)^{2}
\end{aligned}
$$

where $\Delta K$ and $\Delta \sigma$ are the stress intensity factor (SIF) range and the applied stress range, $l$ and $l_{0}$ are the short crack length and El Haddad parameter. $\Delta K_{t h}$ and $\Delta \sigma_{e}$ are the threshold SIF and the fatigue limit of the material at which the small cracks do not propagate.

Murakami [39] adopted a new parameter $\sqrt{\text { area }}$ to obtain the relationship between the SIF range and the porosity size, where the spherical pore is treated as a crack with a size equal to the square root of the projected area of the pore and the SIF range can be estimated in the following form:

$$
\Delta K_{p}=C \Delta \sigma \sqrt{\pi \sqrt{\text { area }}}
$$

The parameter $C$ depends on the porosity defects location, where $C=0.5$ is for internal pores and $C=0.65$ is for external pores [39].

Beretta and Romano [40] expressed El Haddad parameter $l_{0}$ by Murakami's parameter $\sqrt{\text { area }}$ in Eq. (24) to have a smooth transition from the porosity defects to the long cracks, and introducing the SIF limit for the specimens with crack nucleation at the pore (Eq. (25)).

$$
\begin{aligned}
& \sqrt{\text { area }_{0}}=\frac{1}{\pi}\left(\frac{\Delta K_{t h, L C}}{\mathrm{C} \Delta \sigma_{e}}\right)^{2} \\
& \Delta K_{t h}=\Delta K_{t h, L C} \sqrt{\frac{\sqrt{\text { area }}}{\sqrt{\text { area }}+\sqrt{\text { area }}}}
\end{aligned}
$$

$\Delta K_{t h, L C}$ is the threshold SIF range for the long crack and according to the tests in [10] $\Delta K_{t h, L C}=4.5 \mathrm{MPa} \sqrt{m}$ for the AM Ti6Al4V with the intrinsic fatigue limit of $\Delta \sigma_{e}=$ $540 M P a$, when load ratio $R=0.1$.

The fatigue strength in the presence of the small defects can be described by Kitagawa and Takahashi diagram (K-T diagram) [67]. K-T diagram can be presented 
in terms of fatigue limit or SIF in the presence of the porosity defects, and the study in [40] shows its good applicability. To analyse the effect of the porosity on the fatigue life of the sample, Eq. (23) is used to calculate the SIF range $\Delta K_{p}$ for the pores with diameter $0 \leq d_{p} \leq 400 \mu \mathrm{m}$ and Eq. (25) - to find the SIF range limit $\Delta K_{t h, p}$ for specified pores. The calculated $\Delta K_{p}$ and $\Delta K_{t h, p}$ are presented by K-T diagram in Fig. 12.

As discussed in Sect. 2, the evolution law of the remaining life in Eq. (8) is expressed in terms of cyclic bond strain, and following the Paris law the evolution law is presented in the following form:

$\frac{d \lambda(N)}{d N}=-A_{1} \Delta K^{m_{1}}$

Because the SIF range is proportional to the strain, the $\Delta K_{t h, p}$ is used as a SIF limit for crack nucleation phase and the effect of the pore on strain can be introduced in the following equation:

$$
\frac{\varepsilon}{\varepsilon_{p}}=\frac{\Delta K_{t h, p}}{\Delta K_{p}}
$$

And the evolution law of the remaining life will be modified in the following way:

$$
\frac{d \lambda(N)}{d N}=-A_{1} \varepsilon_{p}{ }^{m_{1}}=-A_{1}\left(\varepsilon \frac{\Delta K_{p}}{\Delta K_{t h, p}}\right)^{m_{1}}
$$

in which $\varepsilon_{p}$ is the cyclic strain of the bonds connected to the material point with a specified pore diameter $d_{p}$. In this way, the pores with different diameter can be added in the numerical model, and their effect can be analysed on the nucleation phase of fatigue life.

Murakami's concept of correlation between the geometrical parameter $\sqrt{\text { area }}$ and the threshold SIF is applicable for different shapes of the defects and cracks for the region $\sqrt{\text { area }} \leq 1000 \mu m$ [66]. Moreover, it includes one pore, which is evaluated as the 'fatal' pore with the fatigue fracture nucleation. But the location of the 'fatal' pore in the specimen and the size is very important and plays a significant role in the fatigue life of the sample. Due to this, Sect. 4.4.1. is presenting the PD numerical results of the effect of one pore on the fatigue life of the Ti6Al4V sample.

\subsubsection{Pore size and location effect on fatigue life}

The analysis is performed on the effect of pore diameter and location on the fatigue life of the sample, using the proposed PD fatigue model of porosity implementation, described in Sect. 4.4. The PD study is conducted with the allocation of one 'fatal' pore with a diameter $d_{p}$ at the distance $w_{p}$ from the sample centre line, as shown in Fig. 13. 
The specimen is under tension-tension loading condition with stress amplitude of $\sigma_{a}=$ $270 \mathrm{MPa}$. PD results in Fig. 14, showing that the closer the pore is to the free edge with higher pore diameter, the lower the life of the sample is. It can be seen that the critical pore diameter is around $d_{p}=100 \mu \mathrm{m}$, which means that the samples with pore diameters $d_{p}<100 \mu m$ result in a small decrease of the titanium alloy fatigue strength.

\subsubsection{Fatigue life of low porosity sample}

The experiments [10] present a wide variation of the sample life under the same loading conditions. Moreover, various experimental studies $[8,10,18,27,68]$ have indicated that the lowest fatigue life is noticed when multiple pores are present close to the free surface. The sub-surface pores are the most critical case for damage initiation, as shown as well in Sect.4.4.1. Having this in mind, the PD fatigue numerical model was implemented for a system with multiple pores. Considering the study of the existence of the multiple micro-cracks in the system [47], in this study, it is assumed that if two pores are interacting with each other, meaning if both material points $x_{i}$ and $x_{j}$ are selected for the representation of the pore, where the evaluated by Eq. (27) cyclic strain of the bonds of material points $x_{i}$ and $x_{j}$ will be $\varepsilon_{p i}$ and $\varepsilon_{p j}$, respectively, then the bond $x_{i}-x_{j}$ between two material points will be assigned with $\varepsilon_{p i} \cdot \varepsilon_{p j}$, indicating the amplifying effect on the crack nucleation process. The following concept includes the interaction of the pores and, if the pores are located close to each other, at a distance between them $\leq 450 \mu \mathrm{m}$ (horizon sizes of PD models is $\delta=$ $0.45 \times 10^{-3} \mathrm{~m}$ ), then the faster crack nucleation will be noticed and more significant impact on the fatigue life.

Instead of using the pre-breaking bond model applied in Sect. 4.3, only the SIFs of each pore is evaluated, and Eq. (28) is used for pores representation in a sample. Note that the proposed relationship is applied to all bonds $N_{T(i)}$ for material point $x_{i}$.

Fig. 15 represents the damage maps of three solutions under different loading conditions with the porosity of $P=0.01 \%$ in the samples. The pattern of the damage is similar to the pre-broken bond method, where the damage is initiating at the pores located in the vicinity of the plate edge. The comparison of the numerically predicted fatigue life for porosity values of $P=0.01 \%$ and $P=0.04 \%$ and tests data is presented in Fig. 16. Multiple simulations were performed for the samples with $P=0.04 \%$ and Fig. 16 shows the scatter of the PD results due to the different pore distribution in the sample. The damage initiates at the pore, closest to the sample surface, where the higher diameter of the pores and their interaction showed lower fatigue life, 
summarised in Table 1. The 'fatal' pore is the pore where the crack nucleates and additional contribution to the crack initiation come from secondary pore located at the close proximity.

The proposed approach of pores initiation in the sample provides fatigue life predictions close to the experiments [10]. Moreover, for the porosity value of $P=$ $0.04 \%$, the new peridynamic porosity model performs better than the CDD model.

\section{Discussion}

The distribution, size, shape, location, and density of the process-induced critical defects impact the performance of AM structures. The samples with the similar manufacturing process, microstructures and smooth surface could result in different fatigue life, due to the high dependency on the initial defects size and stress levels, which brings the high scatter of the test fatigue data [11,18-21]. Due to this, it is important to understand the impact of process-induced porocities of fatigue life of AM samples.

The fatigue studies have been conducted for the AM Ti6AI4V under load controlled constant amplitude condition with a load ratio of $R=0.1$. One of the challenges of investigating the AM materials fatigue life is the implementation of the porosity defects in the numerical model. For the numerical purpose, the developed PD fatigue model has been set-up for the defect-free Ti6Al4V sample using the test data for the PD parameters' calibration process. This approach indicated the workability of the developed model, which is named as a reference one. Although several assumptions are made in the PD model, like the material is treated as elastic with isotropic properties even when the pores are initiated in the model, the numerical results showed good fatigue life predictability.

It is also well known that the sample undergoes the cyclic plastic deformations on microscopic levels and controls the material's fatigue life. The cracks are starting to initiate at the share of the persistent slip bands due to the development of stress concentrations [60-62]. On the other hand, the existence of the pores or inclusions is becoming the critical factor for crack initiation. The defects located at the close vicinity to the surface have had the high local stresses and with the inclusions of $d_{p}>10 \mu \mathrm{m}$ originated the crack nucleation [63]. Flat elongated defects have higher stress concentrations and are more critical for the fatigue performance. However, even perfectly round defects have an effect on crack nucleation. Based on these observations in the literature, the phenomena such as stress relaxation, strain 
hardening, phase transformation are excluded from the current PD fatigue model, and the evolution of the crack nucleation is starting at the pore, so the pore is always in the elastic domain, and no plastic flow occurs.

The performed numerical investigation has demonstrated the vital reduction in the fatigue life of the Ti6Al4V dog-bone specimen caused by the presence of the pores in the sample. The early failure in the samples was influenced by a few large pores (with diameter of $d_{p}>100 \mu \mathrm{m}$ ) located close to the surface or the multiple pores located in the close proximity. The CDD approach of porosity implementation results in local stress concentrations at the pre-damaged zone, developing in crack initiation. But the following concept overpredicted the fatigue life of the sample and required further investigation on its applicability on fatigue nucleation problems. However, the adopted Murakami's formulation in the PD fatigue model, the SIF of the pore defect was evaluated with the assumption that all the pores are spherical. The numerical model was simulated for the two types of porosities of $P=0.01 \%$ and $P=0.04 \%$ and the results are quite close to the test results available in literature. Note that the model is treated as elastic and plastic slips and dislocation processes are omitted, where crack nucleation starts directly at the allocated pore in a sample. Nevertheless, the proposed method with the adopted assumptions of pores allocation in the PD model has shown an ability to estimate the most critical defect's size and location.

S-N curves are generated under constant amplitude loading with the load ratio of $R=0.1$, while the loads can often be with variable amplitudes in nature. Under various loading conditions, load sequence can be important. Used linear cumulative damage rule in the PD studies for fatigue life predictions needs to be investigated for AM structures under variable amplitude or under different load ratios. If periodic overloads exist in the structure, then the cycling hardening or softening can occur in the structure. The PD model has to be extended and include both elastic and plastic processes in the material.

The insight provided by the PD analyses can be used for fatigue life predictions considering both internal and surface defects, with the study of different defect shapes, are potential subjects of future works. 
Table 1 PD results of the effect of porosity diameter on fatigue life with $P=0.04 \%$

\begin{tabular}{cccc}
\hline $\begin{array}{c}\text { Stress amplitude } \\
\sigma_{a}, M P a\end{array}$ & $\begin{array}{c}\text { Fatigue life } \\
N_{f}, \text { cycles }\end{array}$ & $\begin{array}{c}\text { 'Fatal' pore } \\
\text { Diameter } d_{p}, \mu m\end{array}$ & $\begin{array}{c}\text { Location } \\
w_{p}, 10^{-3} \mathrm{~m}\end{array}$ \\
\hline 316 & 10894 & 210 & 3 \\
316 & 18053 & 160,35 & $2.85,2.7$ \\
316 & 26418 & 17,124 & $3,2.85$ \\
270 & 83228 & 21,250 & $3,2.85$ \\
270 & 91750 & 162,47 & $3,2.85$ \\
270 & 203853 & 164,60 & $2.85,2.7$ \\
270 & 223221 & 222 & 2.85 \\
270 & 357848 & 55,93 & $3,2.85$ \\
225 & 222554 & 350 & 2.85 \\
225 & 1461034 & 200 & 2.85
\end{tabular}

\section{Conclusion}

In this work, the authors present the results of considering the porosity effect and pore defects on the fatigue nucleation of AM Ti6Al4V material. Those effects were evaluated based on the porosity levels publicly available in the literature. The authors successfully implemented peridynamic theory to model pores in 2-D dog-bone titanium alloy specimen and took into account the interaction between the pores. The work included two types of porosity implementations, where the CDD model was used with pre-broken bonds in the sample, and new peridynamic porosity model with the enforced coefficient in the evolution law of the remaining life of the bonds. The porosity of the sample was accounted for by Gamma distribution, where the identified diameters of the pores were randomly distributed in the samples. Firstly, the CDD approach was applied in the fatigue model, where the fatigue life was overpredicted for the samples with the higher porosities of $P=0.04 \%$. Secondly, the new peridynamic porosity model-based life prediction method demonstrated the improved capability of capturing the effect of both porosities on fatigue nucleation. The presented S-N curves show that the PD fatigue life prediction is in a good agreement with the test data. The authors evaluated the dependency of the fatigue life on pore location and size and showcased that the subsurface pores with the pore diameter are the most critical. The larger pore located in the vicinity of the sample surface has a high contribution to fatigue life reduction. The developed PD fatigue model showed its capability to predict the fatigue life of the defect free-samples, as well as of the samples with different types of porosities. 


\section{References}

[1] W.E. Frazier, Metal additive manufacturing: A review, J. Mater. Eng. Perform. 23 (2014) 19171928. https://doi.org/10.1007/s11665-014-0958-z.

[2] L.E. Murr, E. Martinez, K.N. Amato, S.M. Gaytan, J. Hernandez, D.A. Ramirez, P.W. Shindo, F. Medina, R.B. Wicker, Fabrication of metal and alloy components by additive manufacturing: Examples of 3D materials science, J. Mater. Res. Technol. 1 (2012) 42-54. https://doi.org/10.1016/S2238-7854(12)70009-1.

[3] J.-P. Kruth, M.C. Leu, T. Nakagawa, Progress in Additive Manufacturing and Rapid Prototyping, CIRP Ann. 47 (1998) 525-540. https://doi.org/10.1016/s0007-8506(07)63240-5.

[4] Y. Zhai, D.A. Lados, J.L. Lagoy, Additive Manufacturing: Making imagination the major Limitation, Jom. 66 (2014) 808-816. https://doi.org/10.1007/s11837-014-0886-2.

[5] J.D. Strickland, Applications of Additive Manufacturing in the marine industry, PRADS 2016 Proc. 13th Int. Symp. Pract. Des. Ships Other Float. Struct. (2016). https://doi.org/10.13140/RG.2.2.29930.31685.

[6] S. Chandrasekaran, S. Hari, M. Amirthalingam, Wire arc additive manufacturing of functionally graded material for marine risers, Mater. Sci. Eng. A. 792 (2020) 139530. https://doi.org/10.1016/j.msea.2020.139530.

[7] T. DebRoy, H.L. Wei, J.S. Zuback, T. Mukherjee, J.W. Elmer, J.O. Milewski, A.M. Beese, A. Wilson-Heid, A. De, W. Zhang, Additive manufacturing of metallic components - Process, structure and properties, Prog. Mater. Sci. 92 (2018) 112-224. https://doi.org/10.1016/j.pmatsci.2017.10.001.

[8] S. Tammas-Williams, P.J. Withers, I. Todd, P.B. Prangnell, The Influence of Porosity on Fatigue Crack Initiation in Additively Manufactured Titanium Components, Sci. Rep. 7 (2017) 1-13. https://doi.org/10.1038/s41598-017-06504-5.

[9] E. Wycisk, A. Solbach, S. Siddique, D. Herzog, F. Walther, C. Emmelmann, Effects of defects in laser additive manufactured Ti-6Al-4V on fatigue properties, in: Phys. Procedia, 2014: pp. 371-378. https://doi.org/10.1016/j.phpro.2014.08.120.

[10] R. Biswal, X. Zhang, A.K. Syed, M. Awd, J. Ding, F. Walther, S. Williams, Criticality of porosity defects on the fatigue performance of wire + arc additive manufactured titanium alloy, Int. J. Fatigue. 122 (2019) 208-217. https://doi.org/10.1016/j.ijfatigue.2019.01.017.

[11] R. Biswal, A.K. Syed, X. Zhang, Assessment of the effect of isolated porosity defects on the fatigue performance of additive manufactured titanium alloy, Addit. Manuf. 23 (2018) 433-442. https://doi.org/10.1016/j.addma.2018.08.024.

[12] M. Seifi, A. Salem, D. Satko, J. Shaffer, J.J. Lewandowski, Defect distribution and microstructure heterogeneity effects on fracture resistance and fatigue behavior of EBM Ti-6Al-4V, Int. J. Fatigue. 94 (2017) 263-287. https://doi.org/10.1016/j.ijfatigue.2016.06.001.

[13] Y. Kok, X.P. Tan, P. Wang, M.L.S. Nai, N.H. Loh, E. Liu, S.B. Tor, Anisotropy and heterogeneity of microstructure and mechanical properties in metal additive manufacturing: A critical review, Mater. Des. 139 (2018) 565-586. https://doi.org/10.1016/j.matdes.2017.11.021.

[14] T. Mukherjee, J.S. Zuback, A. De, T. Debroy, Printability of alloys for additive manufacturing, Sci. Rep. 6 (2016) 19717. https://doi.org/10.1038/srep19717.

[15] G. Kasperovich, J. Hausmann, Improvement of fatigue resistance and ductility of TiAl6V4 
processed by selective laser melting, J. Mater. Process. Technol. 220 (2015) 202-214. https://doi.org/10.1016/j.jmatprotec.2015.01.025.

[16] F. Martina, P.A. Colegrove, S.W. Williams, J. Meyer, Microstructure of Interpass Rolled Wire + Arc Additive Manufacturing Ti-6Al-4V Components, Metall. Mater. Trans. A Phys. Metall. Mater. Sci. 46 (2015) 6103-6118. https://doi.org/10.1007/s11661-015-3172-1.

[17] L. Thijs, F. Verhaeghe, T. Craeghs, J. Van Humbeeck, J.P. Kruth, A study of the microstructural evolution during selective laser melting of Ti-6Al-4V, Acta Mater. 58 (2010) 3303-3312. https://doi.org/10.1016/j.actamat.2010.02.004.

[18] E. Wycisk, S. Siddique, D. Herzog, F. Walther, C. Emmelmann, Fatigue performance of laser additive manufactured Ti-6Al-4V in very high cycle fatigue regime up to 109 cycles, Front. Mater. 2 (2015) 2-9. https://doi.org/10.3389/fmats.2015.00072.

[19] S. Leuders, M. Thöne, A. Riemer, T. Niendorf, T. Tröster, H.A. Richard, H.J. Maier, On the mechanical behaviour of titanium alloy TiAl6V4 manufactured by selective laser melting: Fatigue resistance and crack growth performance, Int. J. Fatigue. 48 (2013) 300-307. https://doi.org/10.1016/j.ijfatigue.2012.11.011.

[20] J. Günther, D. Krewerth, T. Lippmann, S. Leuders, T. Tröster, A. Weidner, H. Biermann, T. Niendorf, Fatigue life of additively manufactured Ti-6Al-4V in the very high cycle fatigue regime, Int. J. Fatigue. 94 (2017) 236-245. https://doi.org/10.1016/j.ijfatigue.2016.05.018.

[21] B. Baufeld, E. Brandl, O. Van Der Biest, Wire based additive layer manufacturing: Comparison of microstructure and mechanical properties of Ti-6Al-4V components fabricated by laser-beam deposition and shaped metal deposition, J. Mater. Process. Technol. 211 (2011) 1146-1158. https://doi.org/10.1016/j.jmatprotec.2011.01.018.

[22] D. Greitemeier, F. Palm, F. Syassen, T. Melz, Fatigue performance of additive manufactured TiAl6V4 using electron and laser beam melting, Int. J. Fatigue. 94 (2017) 211-217. https://doi.org/10.1016/j.ijfatigue.2016.05.001.

[23] A.B. Spierings, T.L. Starr, K. Wegener, Fatigue performance of additive manufactured metallic parts, Rapid Prototyp. J. 19 (2013) 88-94. https://doi.org/10.1108/13552541311302932.

[24] Q.C. Liu, J. Elambasseril, S.J. Sun, M. Leary, M. Brandt, P.K. Sharp, The effect of manufacturing defects on the fatigue behaviour of Ti-6AI-4V specimens fabricated using selective laser melting, Adv. Mater. Res. 891-892 (2014) 1519-1524. https://doi.org/10.4028/www.scientific.net/AMR.891-892.1519.

[25] E. Brandl, U. Heckenberger, V. Holzinger, D. Buchbinder, Additive manufactured AISi10Mg samples using Selective Laser Melting (SLM): Microstructure, high cycle fatigue, and fracture behavior, Mater. Des. 34 (2012) 159-169. https://doi.org/10.1016/j.matdes.2011.07.067.

[26] A. Fatemi, R. Molaei, S. Sharifimehr, N. Phan, N. Shamsaei, Multiaxial fatigue behavior of wrought and additive manufactured Ti-6AI-4V including surface finish effect, Int. J. Fatigue. 100 (2017) 347-366. https://doi.org/10.1016/j.ijfatigue.2017.03.044.

[27] X. Liu, C. Sun, Y. Hong, Effects of stress ratio on high-cycle and very-high-cycle fatigue behavior of a Ti-6Al-4V alloy, Mater. Sci. Eng. A. 622 (2015) 228-235. https://doi.org/10.1016/j.msea.2014.09.115.

[28] P.A. Kobryn, E.H. Moore, S.L. Semiatin, Effect of laser power and traverse speed on microstructure, porosity, and build height in laser-deposited Ti-6Al-4V, Scr. Mater. 43 (2000) 299-305. https://doi.org/10.1016/S1359-6462(00)00408-5. 
[29] S.D. BROWN, R.B. BIDDULPH, P.D. WILCOX, A Strength-Porosity Relation Involving Different Pore Geometry and Orientation, J. Am. Ceram. Soc. 47 (1964) 320-322. https://doi.org/10.1111/j.1151-2916.1964.tb12993.x.

[30] A.R. Boccaccini, G. Ondracek, P. Mazilu, D. Windelberq, On the effective young's modulus of elasticity for porous materials: Microstructure modelling and comparison between calculated and experimental values, Boccaccini, A. R., Ondracek, G. (1993). Eff. Young's Modul. Elast. Porous Mater. Microstruct. Model. Comp. Between Calc. Exp. Values. 4.4 (1993) 119-128. https://doi.org/10.1515/JMBM.1993.4.2.119.

[31] B. Paul, The Elastic Moduli of Heterogeneous Materials, (1962).

[32] Z. Hashin, The elastic moduli of heterogeneous materials, 1960. https://doi.org/10.1115/1.3636446.

[33] F.P. KNUDSEN, Dependence of Mechanical Strength of Brittle Polycrystalline Specimens on Porosity and Grain Size, J. Am. Ceram. Soc. 42 (1959) 376-387. https://doi.org/10.1111/j.11512916.1959.tb13596.x.

[34] L.F. Nielsen, Strength and Stiffness of Porous Materials, J. Am. Ceram. Soc. 73 (1990) 26842689. https://doi.org/10.1111/j.1151-2916.1990.tb06746.x.

[35] K.K. Phani, S.K. Niyogi, Young's modulus of porous brittle solids, J. Mater. Sci. 22 (1987) 257263. https://doi.org/10.1007/BF01160581.

[36] W. Pabst, E. Gregorová, New relation for the porosity dependence of the effective tensile modulus of brittle materials, J. Mater. Sci. $39 \quad$ (2004) 3501-3503. https://doi.org/10.1023/B:JMSC.0000026961.12735.2a.

[37] J. Kováčik, Correlation between Poisson's ratio and porosity in porous materials, J. Mater. Sci. 41 (2006) 1247-1249. https://doi.org/10.1007/s10853-005-4237-0.

[38] M.H. El Haddad, T.H. Topper, K.. Smith, Prediction of non propagating cracks, Eng. Fract. Mech. 11 (1978) 573-584.

[39] Y. Murakami, Effects of small defects and nonmetallic inclusions on the fatigue strength of metals, Mech. Corros. Prop. Ser. A, Key Eng. Mater. 51-52 (1991) 37-42. https://doi.org/10.4028/www.scientific.net/kem.51-52.37.

[40] S. Beretta, S. Romano, A comparison of fatigue strength sensitivity to defects for materials manufactured by AM or traditional processes, Int. J. Fatigue. 94 (2017) 178-191. https://doi.org/10.1016/j.ijfatigue.2016.06.020.

[41] L. Sheridan, O.E. Scott-Emuakpor, T. George, J.E. Gockel, Relating porosity to fatigue failure in additively manufactured alloy 718, Mater. Sci. Eng. A. 727 (2018) 170-176. https://doi.org/10.1016/j.msea.2018.04.075.

[42] S. Siddique, M. Imran, M. Rauer, M. Kaloudis, E. Wycisk, C. Emmelmann, F. Walther, Computed tomography for characterization of fatigue performance of selective laser melted parts, Mater. Des. 83 (2015) 661-669. https://doi.org/10.1016/j.matdes.2015.06.063.

[43] S.A. Silling, Reformulation of elasticity theory for discontinuities and long-range forces, J. Mech. Phys. Solids. 48 (2000) 175-209. https://doi.org/10.1016/S0022-5096(99)00029-0.

[44] S.A. Silling, O. Weckner, E. Askari, F. Bobaru, Crack nucleation in a peridynamic solid, Int. J. Fract. 162 (2010) 219-227. https://doi.org/10.1007/s10704-010-9447-z.

[45] D. Huang, G. Lu, P. Qiao, An improved peridynamic approach for quasi-static elastic deformation and brittle fracture analysis, Int. J. Mech. Sci. 94-95 (2015) 111-122. 
https://doi.org/10.1016/j.ijmecsci.2015.02.018.

[46] M.F. Basoglu, Z. Zerin, A. Kefal, E. Oterkus, A computational model of peridynamic theory for deflecting behavior of crack propagation with micro-cracks, Comput. Mater. Sci. 162 (2019) 3346. https://doi.org/10.1016/j.commatsci.2019.02.032.

[47] O. Karpenko, S. Oterkus, E. Oterkus, Influence of Different Types of Small-Size Defects on Propagation of Macro-cracks in Brittle Materials, J. Peridynamics Nonlocal Model. 2 (2020) 289316. https://doi.org/10.1007/s42102-020-00032-z.

[48] E. Oterkus, I. Guven, E. Madenci, Fatigue failure model with peridynamic theory, in: 2010 12th IEEE Intersoc. Conf. Therm. Thermomechanical Phenom. Electron. Syst., IEEE, 2010. https://doi.org/10.1109/itherm.2010.5501273.

[49] S. Silling, A. Askari, Peridynamic model for fatigue cracks, 2014. http://docs.lib.purdue.edu/ses2014/mss/cfm/22/\%0Ahttps://www.osti.gov/scitech/biblio/116028 9-peridynamic-model-fatigue-cracking.

[50] G. Zhang, Q. Le, A. Loghin, A. Subramaniyan, F. Bobaru, Validation of a peridynamic model for $\begin{array}{llllll}\text { fatigue cracking, } & \text { Eng. } & \text { Fract. Mech. } & 162 & \text { (2016) }\end{array}$ https://doi.org/10.1016/j.engfracmech.2016.05.008.

[51] Y.L. Hu, E. Madenci, Peridynamics for fatigue life and residual strength prediction of composite $\begin{array}{lllll}\text { laminates, } & \text { Compos. } & \text { Struct. } & 160 & \text { (2017) }\end{array}$ https://doi.org/10.1016/j.compstruct.2016.10.010.

[52] Z. Chen, F. Bobaru, Peridynamic modeling of pitting corrosion damage, J. Mech. Phys. Solids. 78 (2015) 352-381. https://doi.org/10.1016/j.jmps.2015.02.015.

[53] B. Kilic, E. Madenci, An adaptive dynamic relaxation method for quasi-static simulations using the peridynamic theory, Theor. Appl. Fract. Mech. 53 (2010) 194-204. https://doi.org/10.1016/j.tafmec.2010.08.001.

[54] E. Madenci, E. Oterkus, ErdogannMadencii- ErkannOterkus Peridynamic Theory and Its Applications, Springer Science+Business Media New York, 2014.

[55] S.A. Silling, E. Askari, A meshfree method based on the peridynamic model of solid mechanics, Comput. Struct. 83 (2005) 1526-1535. https://doi.org/10.1016/j.compstruc.2004.11.026.

[56] S.A. Silling, F. Bobaru, Peridynamic modeling of membranes and fibers, Int. J. Non. Linear. Mech. 40 (2005) 395-409. https://doi.org/10.1016/j.jijnonlinmec.2004.08.004.

[57] C. Diyaroglu, Peridynamics and its applications in marine structures, University of Strathclyde, 2016.

[58] X. Zhao, S. Li, M. Zhang, Y. Liu, T.B. Sercombe, S. Wang, Y. Hao, R. Yang, L.E. Murr, Comparison of the microstructures and mechanical properties of Ti-6Al-4V fabricated by selective laser melting and electron beam melting, Mater. Des. 95 (2016) 21-31. https://doi.org/10.1016/j.matdes.2015.12.135.

[59] F. Bobaru, M. Yang, L. Frota Alves, S.A. Silling, E. Askari, J. Xu, Convergence, adaptive refinement, and scaling in 1D peridynamics, Int. J. Numer. Methods Eng. (2008). https://doi.org/10.1002/nme.

[60] X. Wu, On Tanaka-Mura's fatigue crack nucleation model and validation, Fatigue Fract. Eng. Mater. Struct. 41 (2018) 894-899. https://doi.org/10.1111/ffe.12736.

[61] W.E. Duckworth, E. Ineson, The effects of externally introduced alumina particles on the fatigue life of En24 steel, Iron Steel Inst. 77 (1963) 87-103. 
[62] C.Y. Kung, M.E. Fine, Fatigue crack initiation and microcrack growth in 2024-T4 and 2124-T4 Aluminium Alloys, Metall. Trans. A. 10A (1979) 603-610. https://doi.org/10.1111/j.14602695.2006.01088.x.

[63] Y. Murakami, K.J. Miller, What is fatigue damage? a view point from the observation of low cycle fatigue process, Int. J. Fatigue. $27 \quad$ (2005) 991-1005. https://doi.org/10.1016/j.jpfatigue.2004.10.009.

[64] Z. Chen, S. Niazi, F. Bobaru, A peridynamic model for brittle damage and fracture in porous $\begin{array}{llllllll}\text { materials, Int. J. Rock Mech. Min. Sci. } 122 & \text { (2019) } & 104059 .\end{array}$ https://doi.org/10.1016/j.jijrmms.2019.104059.

[65] H.D. Carlton, A. Haboub, G.F. Gallegos, D.Y. Parkinson, A.A. MacDowell, Damage evolution and failure mechanisms in additively manufactured stainless steel, Mater. Sci. Eng. A. 651 (2016) 406-414. https://doi.org/10.1016/j.msea.2015.10.073.

[66] Y. Murakami, M. Endo, Effects of defects, inclusions and inhomogeneities on fatigue strength, Int. J. Fatigue. 16 (1994) 163-182. https://doi.org/10.1016/0142-1123(94)90001-9.

[67] S. Kitagawa, H., Takahashi, Application of fracture mechanics to very small cracks or the cracks in early stage, in: Proc. 2nd ICM, Clevel., 1976: pp. 627-631.

[68] F. Fomin, M. Horstmann, N. Huber, N. Kashaev, Probabilistic fatigue-life assessment model for laser-welded Ti-6Al-4V butt joints in the high-cycle fatigue regime, Int. J. Fatigue. 116 (2018) 22-35. https://doi.org/10.1016/j.ijfatigue.2018.06.012. 


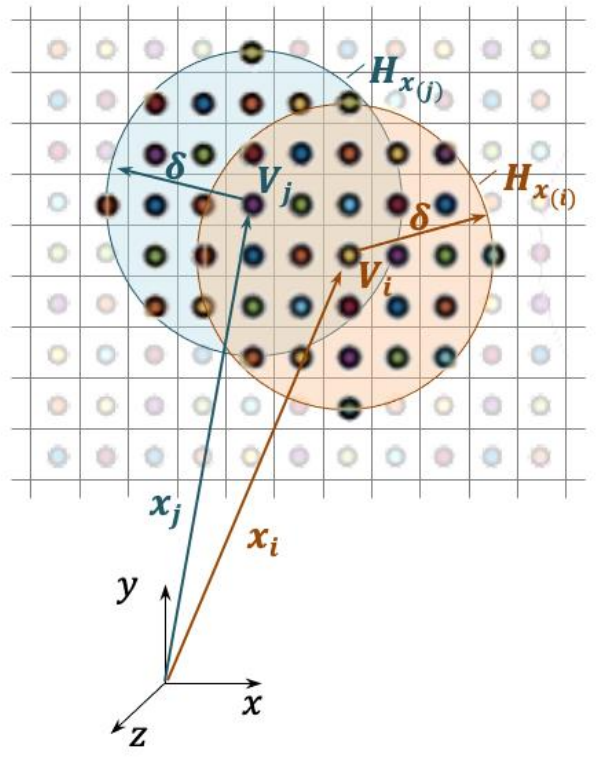

Fig. 1 Peridynamic material points and interaction of material points $i$ and $j$.

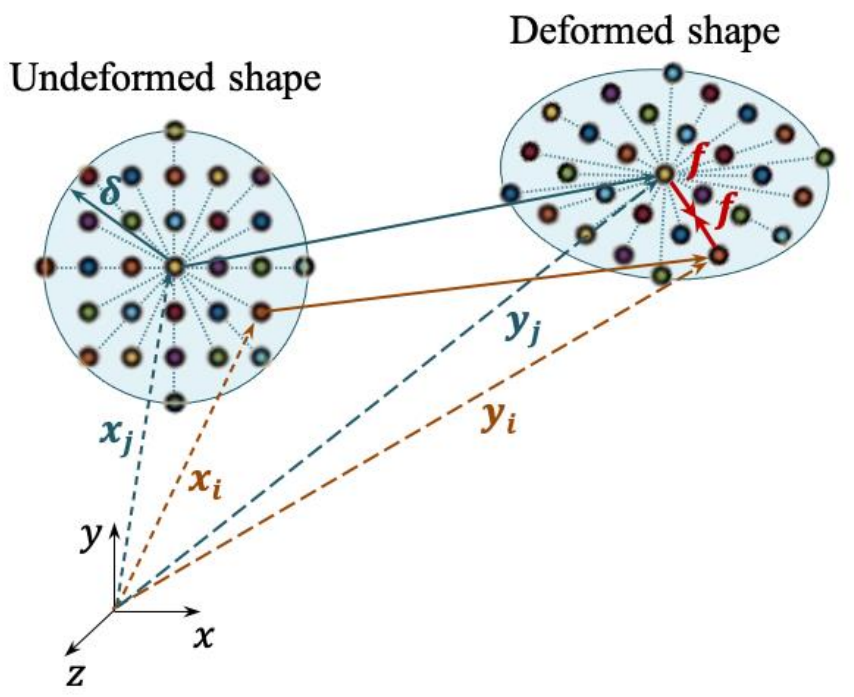

Fig. 2 Peridynamic material points $i$ and $j$ in the deformed configuration and the peridynamic forces between these material points. 
$\mathbf{a}$

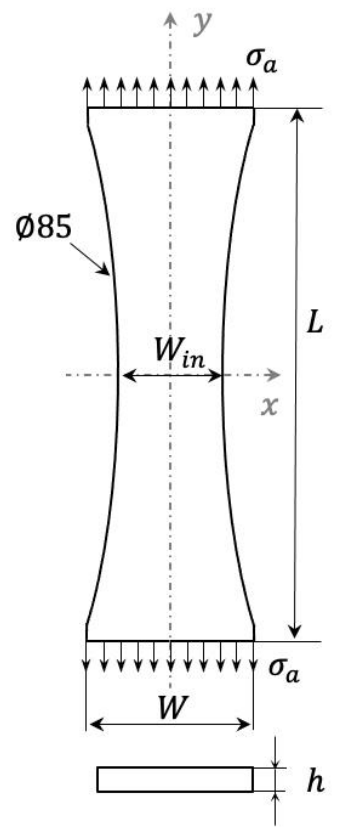

b

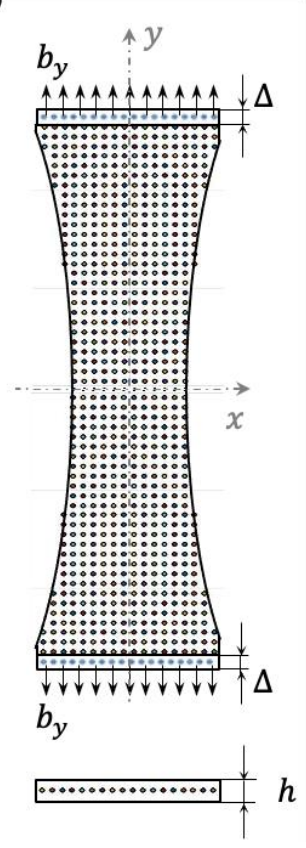

Fig. 3 a Dog-bone titanium alloy plate under uniaxial tensile loading and $\mathbf{b}$ its discretisation.

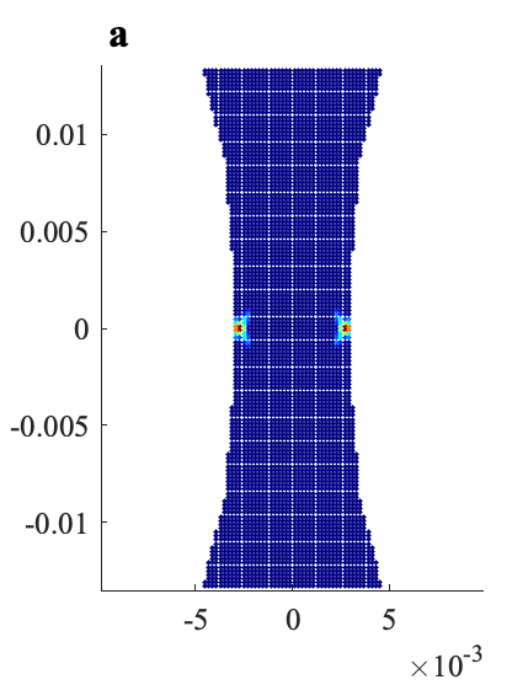

b

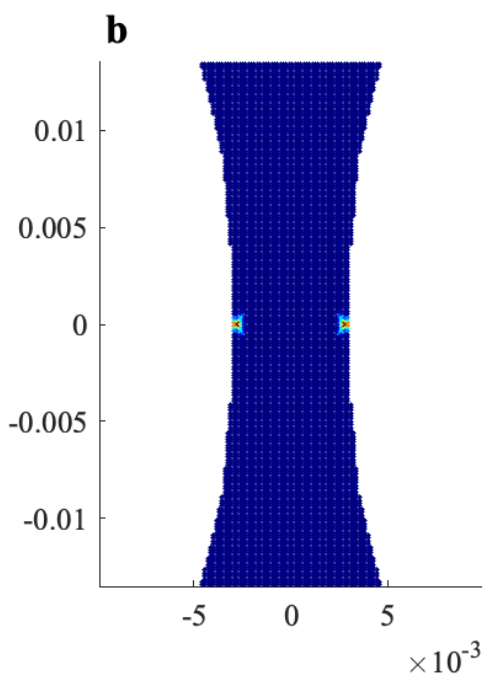

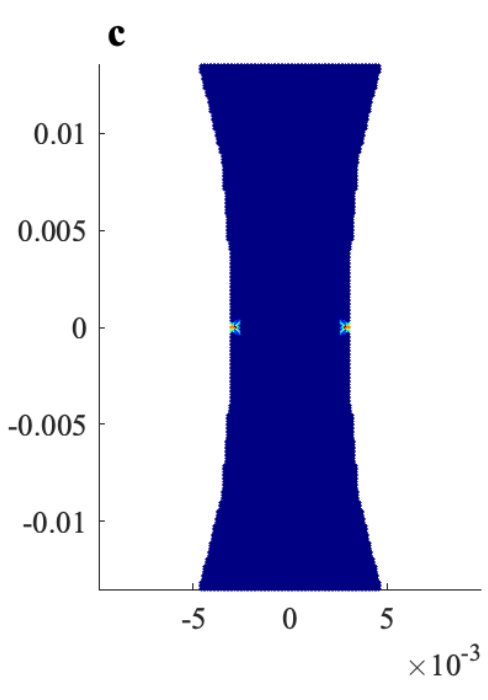

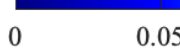

0.1

0.15

0.2

0.25

0.3

0.35

0.4

Fig. $4 \delta$ - convergence of PD fatigue crack nucleation for three different horizon sizes and the corresponding predicted life $N_{f}$ : a $\delta=0.6 \times 10^{-3} \mathrm{~m}, N_{f}=194571$ cycle; b $\delta=0.45 \times 10^{-3} \mathrm{~m}, N_{f}=$ 194059 cycles; c $\delta=0.3 \times 10^{-3} \mathrm{~m}, N_{f}=193240$ cycles 


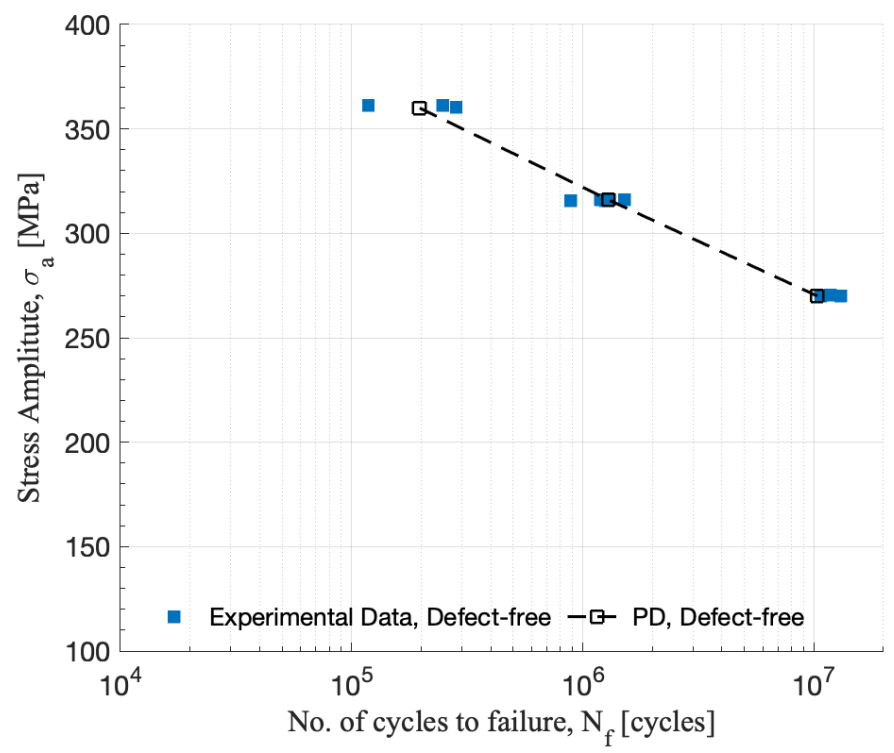

Fig. 5 Stress amplitude as a function of the loading cycle at which nucleation occurs

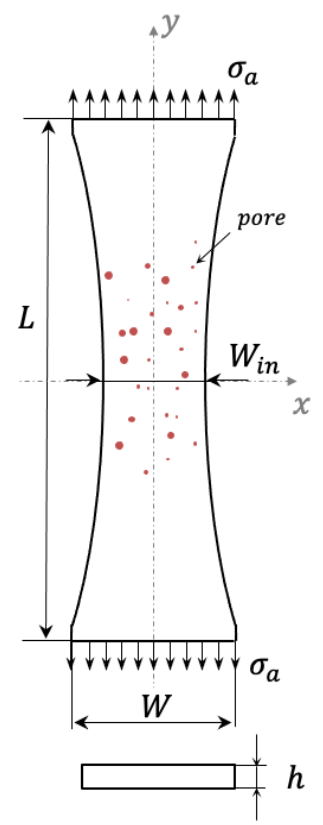

Fig. 6 Sample geometry with randomly distributed pores. 


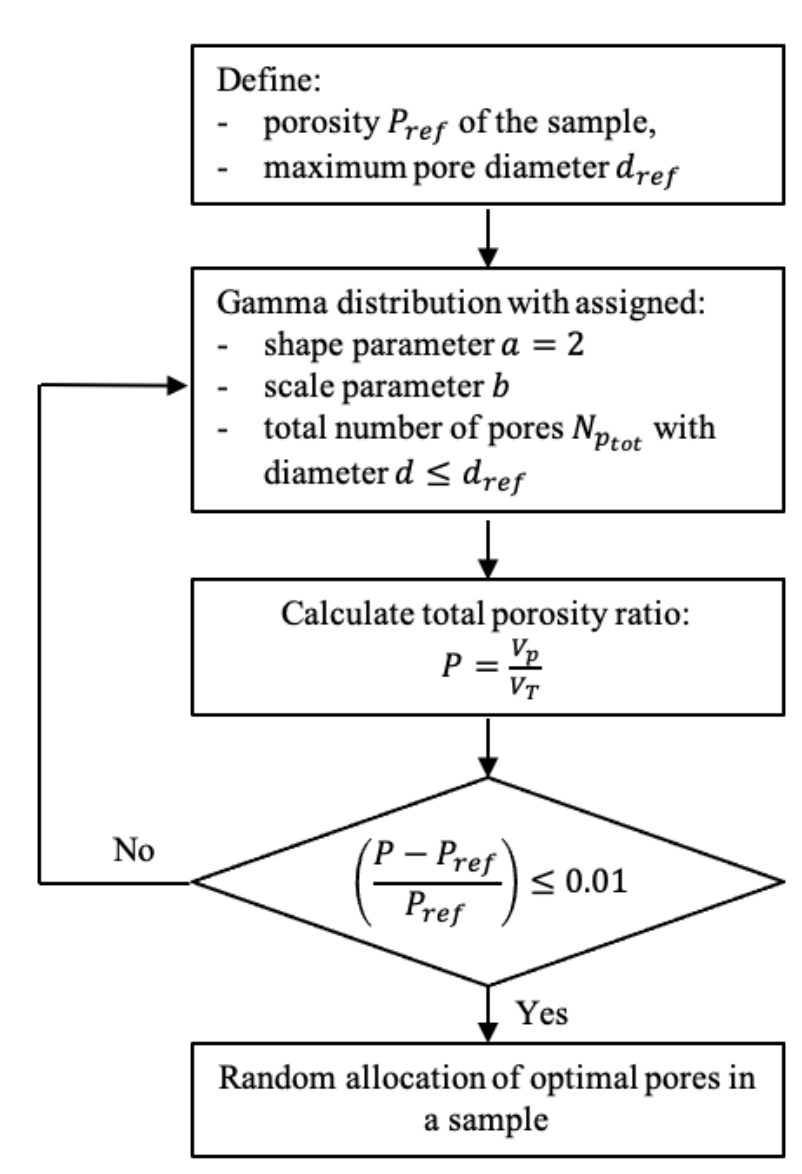

Fig. 7 Flow-chart of porosity evaluation.

a

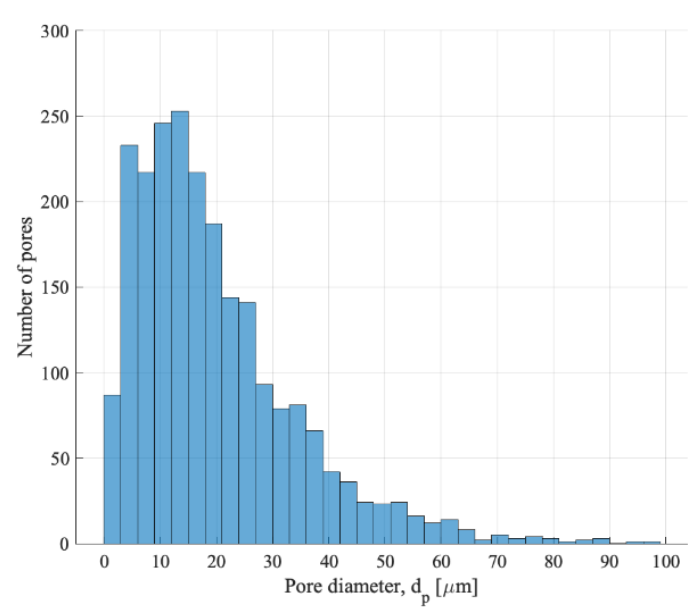

b

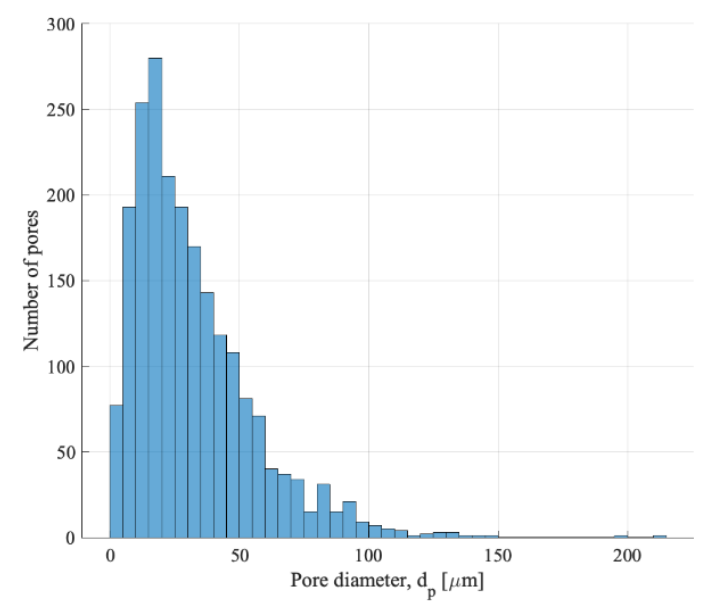

Fig. 8 Gamma distribution: a $a=2, b=10, N_{p_{\text {tot }}}=2256 \rightarrow P=0.01 \%$; b $a=2, b=15, N_{p_{\text {tot }}}=$ $2488 \rightarrow P=0.04 \%$. 


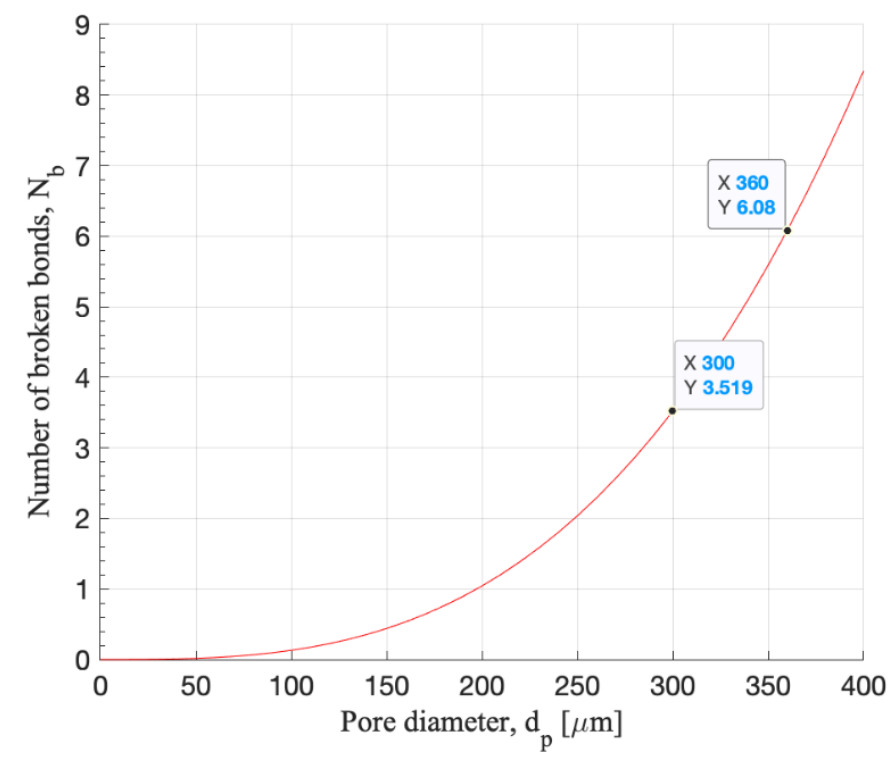

Fig. 9 Pore diameter and the number of broken bonds relationship.
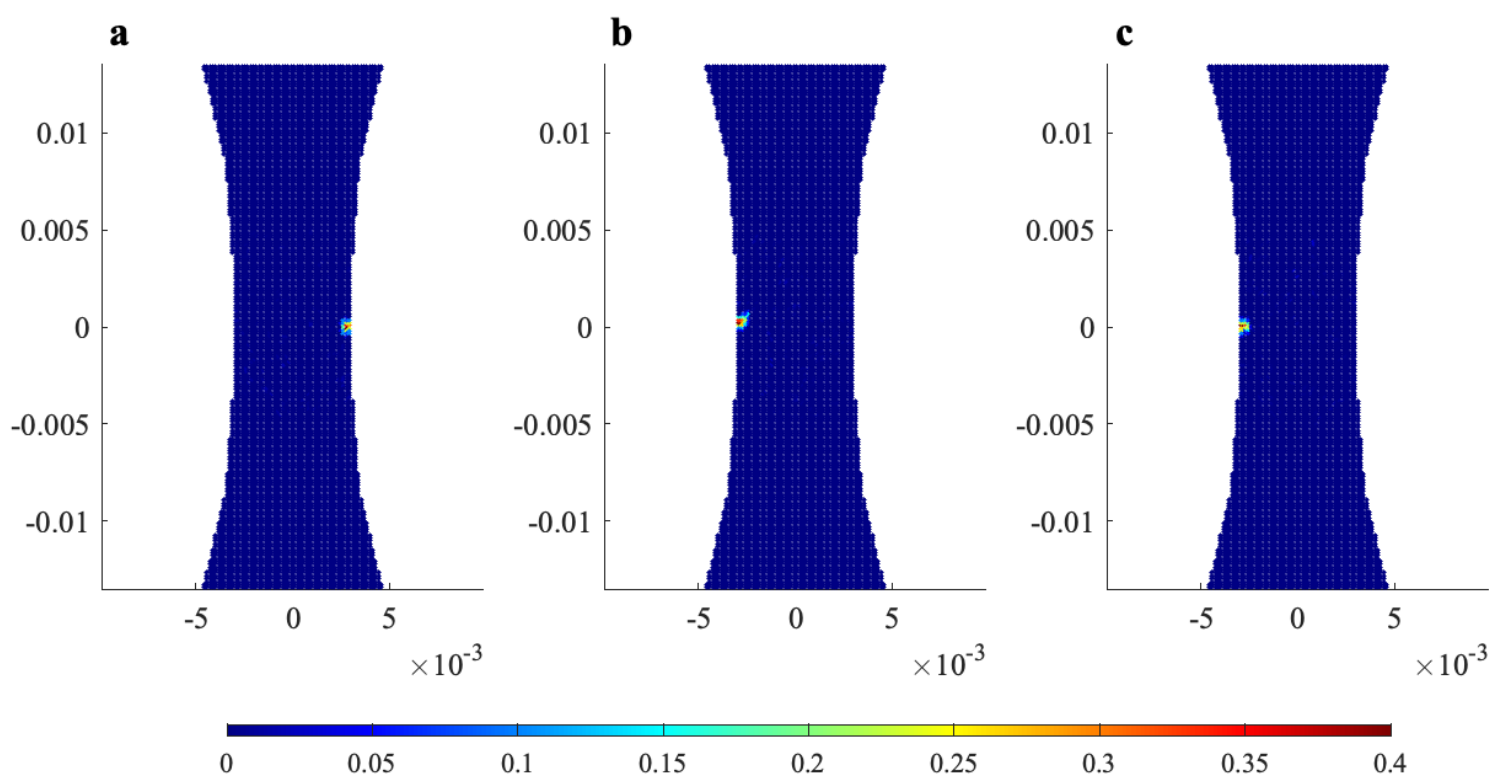

Fig. 10 Damage maps: a $\sigma_{a}=360 M P a, N_{f}=441174$ cycles; $\mathbf{b} \sigma_{a}=270 M P a, N_{f}=910241$ cycles; c $\sigma_{a}=225 \mathrm{MPa}, N_{f}=9837299$ cycles

a

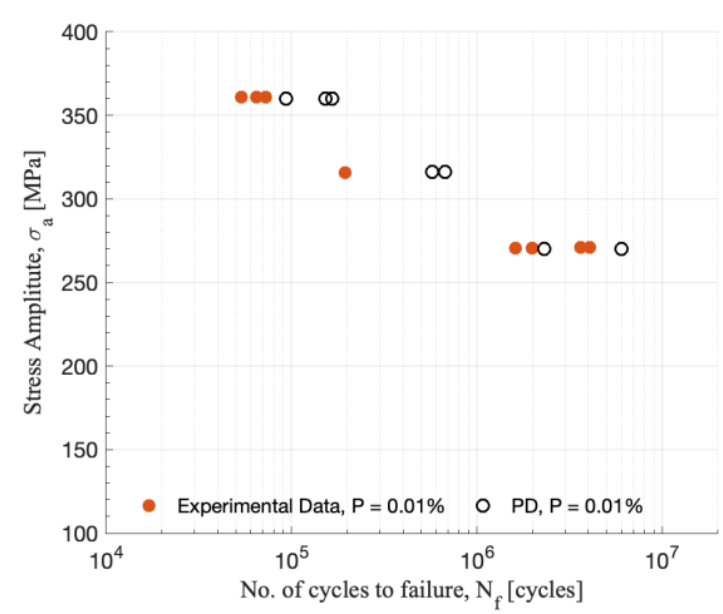

b

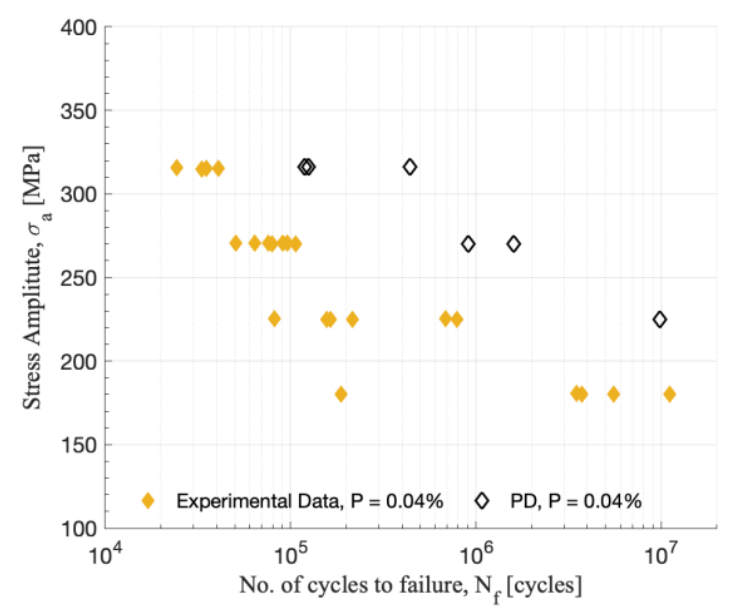


C

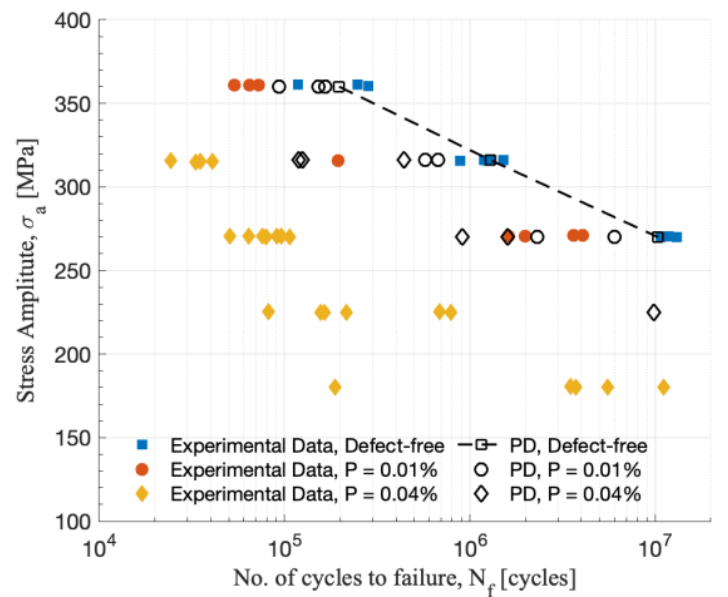

Fig. 11 Experimental and PD results of fatigue life prediction for porosities: a $P=0.01 \%$; $\mathbf{b} P=$ $0.04 \%$ c Combined results for all three models

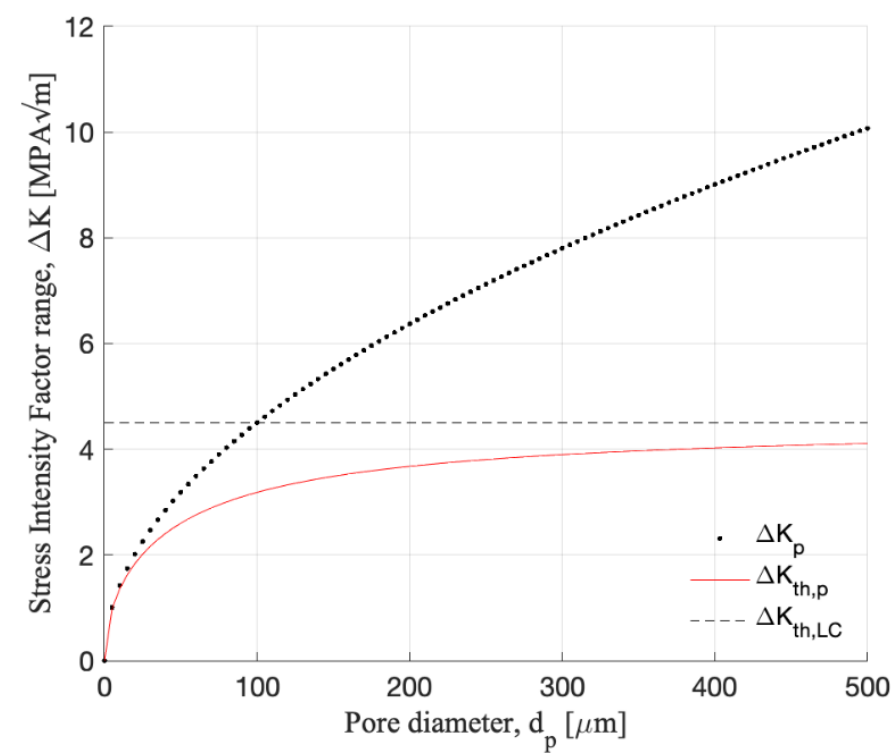

Fig. $12 \mathrm{~K}-\mathrm{T}$ diagram for Ti6Al4V alloy

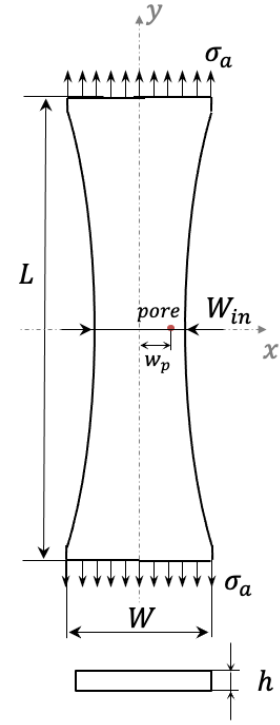

Fig. 13 Sample geometry with the allocation of one pore. 


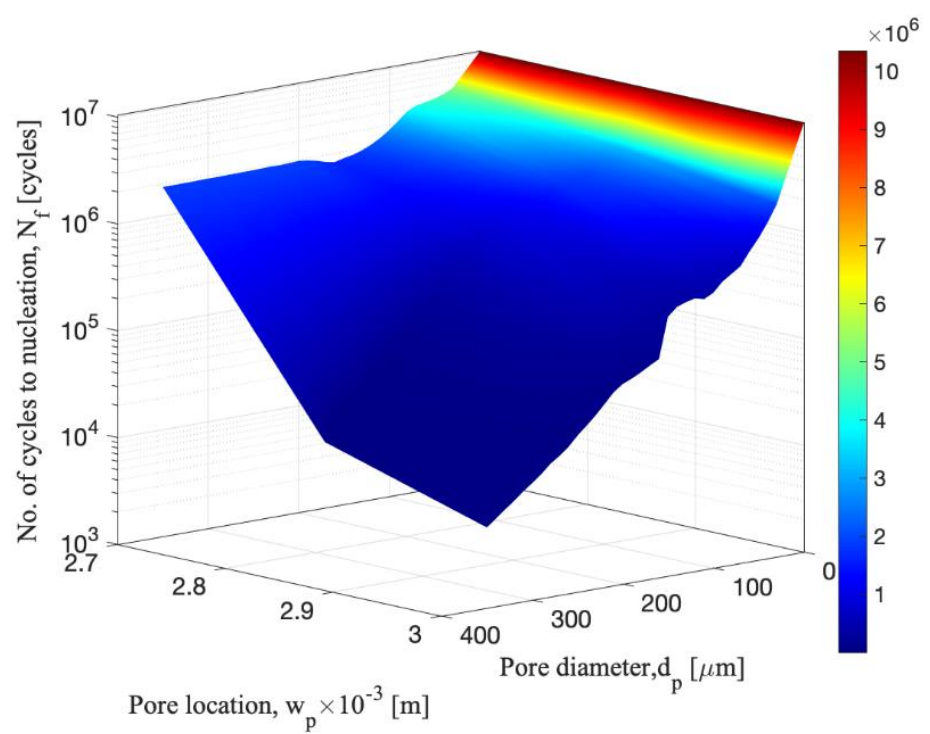

Fig. 14 Effect of pore location and diameter on fatigue life.

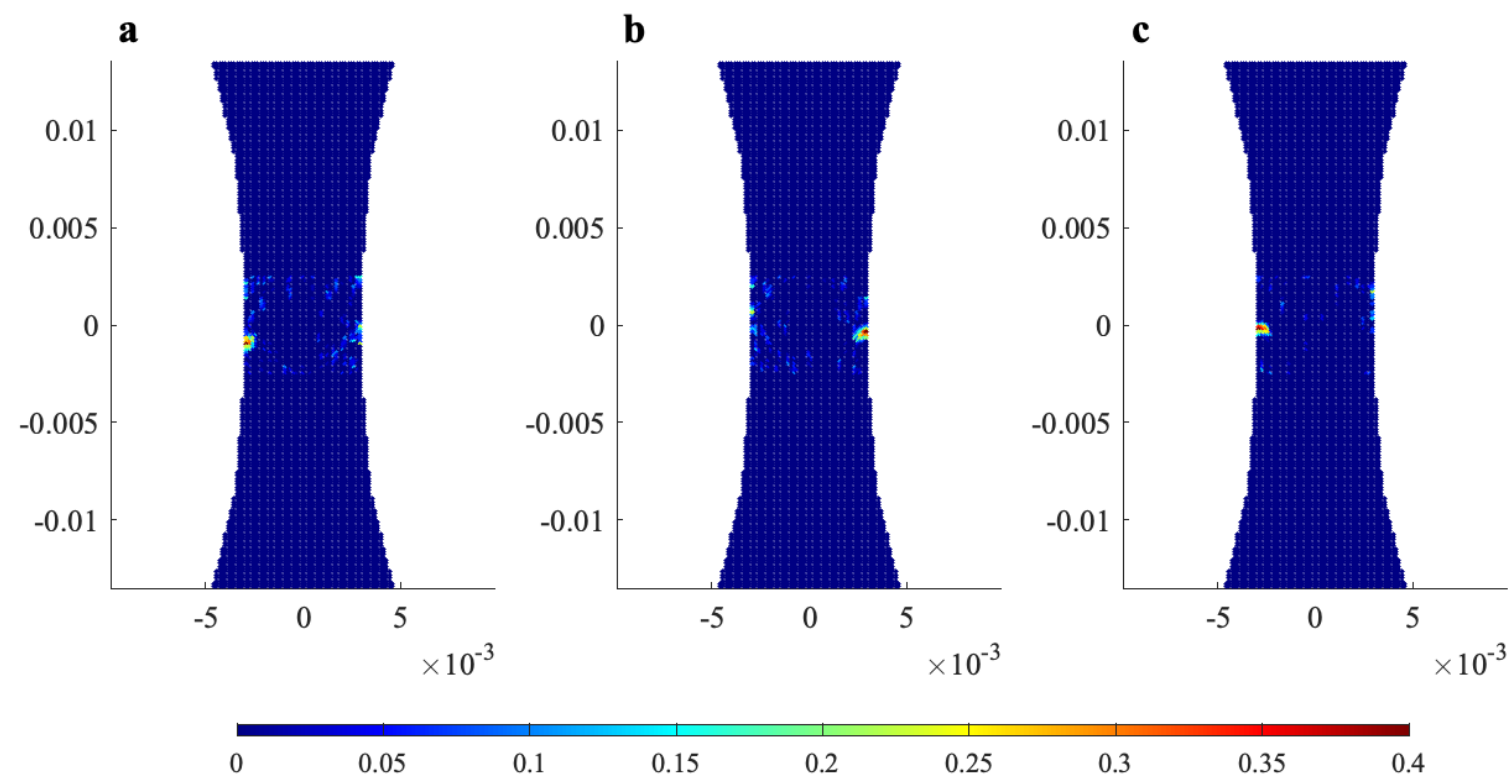

Fig. 15 Damage maps for samples with $P=0.01 \%$ : $\mathbf{a} \sigma_{a}=360 \mathrm{MPa}, N_{f}=33135$ cycles; $\mathbf{b} \sigma_{a}=$ $316 \mathrm{MPa}, N_{f}=130872$ cycles; $\mathbf{c} \sigma_{a}=270 \mathrm{MPa}, N_{f}=1297377$ cycles.

a

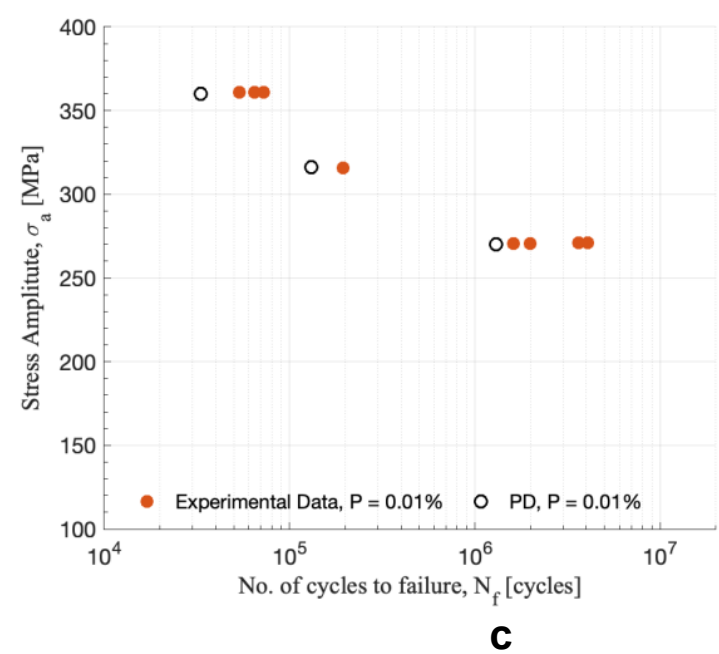

b

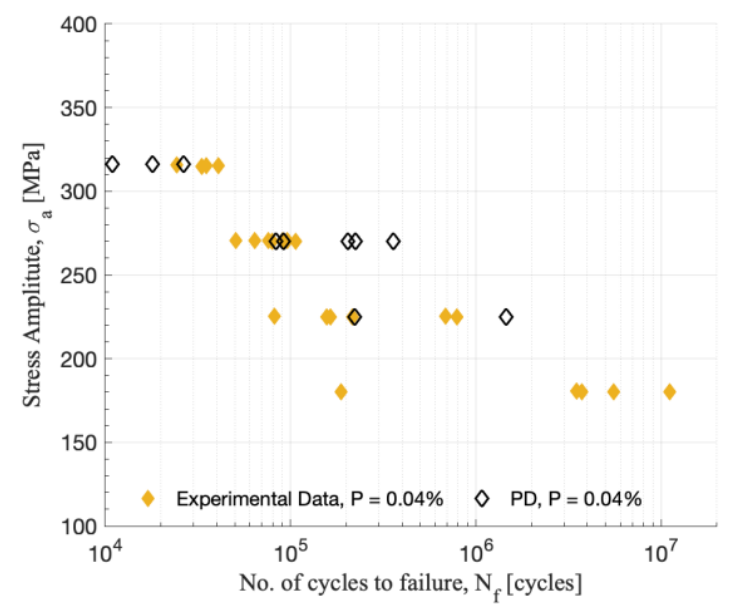




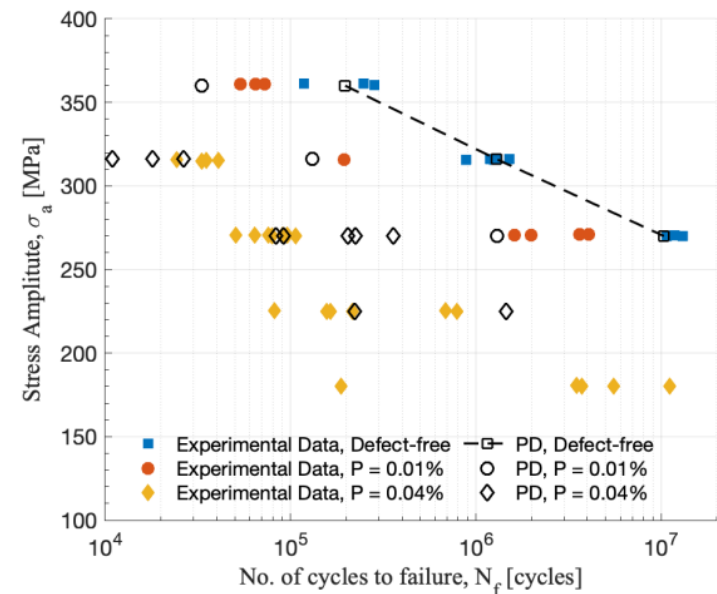

Fig. 16 Experimental and PD results of fatigue life prediction for porosities: a $P=0.01 \%$; $\mathbf{b} P=$ $0.04 \% \mathrm{c}$ Combined results for all three models 